\title{
Osa-miR7695 enhances transcriptional priming in defense responses against the rice blast fungus
}

Ferran Sánchez-Sanuy ${ }^{1}$, Cristina Peris-Peris ${ }^{1}$, Shiho Tomiyama ${ }^{2}$, Kazunori Okada ${ }^{2}$, Yue-le Hsing ${ }^{3}$, Blanca San Segundo ${ }^{1,4}$ and Sonia Campo ${ }^{1 *}$ (i)

\begin{abstract}
Background: MicroRNAs (miRNAs) are small non-coding RNAs that regulate gene expression at the posttranscriptional level in eukaryotes. In rice, MIR7695 expression is regulated by infection with the rice blast fungus Magnaporthe oryzae with subsequent down-regulation of an alternatively spliced transcript of natural resistanceassociated macrophage protein 6 (OsNramp6). NRAMP6 functions as an iron transporter in rice.

Results: Rice plants grown under high iron supply showed blast resistance, which supports that iron is a factor in controlling blast resistance. During pathogen infection, iron accumulated in the vicinity of $M$. oryzae appressoria, the sites of pathogen entry, and in cells surrounding infected regions of the rice leaf. Activation-tagged MIR7695 rice plants (MIR7695-Ac) exhibited enhanced iron accumulation and resistance to M. oryzae infection. RNA-seq analysis revealed that blast resistance in MIR7695-Ac plants was associated with strong induction of defense-related genes, including pathogenesis-related and diterpenoid biosynthetic genes. Levels of phytoalexins during pathogen infection were higher in MIR7695-Ac than wild-type plants. Early phytoalexin biosynthetic genes, OsCPS2 and OsCPS4, were also highly upregulated in wild-type rice plants grown under high iron supply.

Conclusions: Our data support a positive role of miR7695 in regulating rice immunity that further underpin links between defense and iron signaling in rice. These findings provides a basis to better understand regulatory mechanisms involved in rice immunity in which miR7695 participates which has a great potential for the development of strategies to improve blast resistance in rice.
\end{abstract}

Keywords: Blast, Defense, Iron, Magnaporthe oryzae, microRNA, miR7695, Oryza sativa, Phytoalexins, Rice, Transcriptomics

\section{Background}

Plants have a sophisticated innate immune system for protection against pathogen infection $[1,2]$. The activation of plant defense responses against pathogens occurs via the recognition of conserved pathogen-associated molecular patterns (PAMPs; previously known as elicitors) by host pattern-recognition receptors (PRR) which, in turn, triggers a signaling cascade leading to the activation of defenserelated responses. Pathogen-induced defense responses include the production of reactive oxygen species (ROS),

\footnotetext{
* Correspondence: sonia.campo@cragenomica.es

${ }^{1}$ Centre for Research in Agricultural Genomics (CRAG) CSIC-IRTA-UAB-UB, Campus Universitat Autònoma de Barcelona (UAB), Bellaterra (Cerdanyola del Vallés), Barcelona, Spain

Full list of author information is available at the end of the article
}

the activation of protein phosphorylation/dephosphorylation cascades, and the production of pathogenesis-related (PR) proteins, among others [3, 4]. Successful pathogens, however, have developed countermeasures to suppress this basal defense in certain plant species and promote disease by delivering effectors into the host. Plants have also evolved Resistance (R) genes that recognize microbial effectors to activate a much stronger immune response, the so called effector-triggered immunity [5]. PTI and ETI have long been considered protein-based mechanisms. However, increasing evidence supports that microRNAs (miRNAs) are also important players in both PTI and ETI [6-11].

MiRNAs are small noncoding RNAs that modulate gene expression in eukaryotes by triggering sequence- 
specific cleavage or translational repression of target genes [12]. Plant miRNAs play a crucial role in the control of developmental processes and adaptation to environmental stresses, both abiotic and biotic stresses [13-16]. Although numerous miRNAs have been reported to be regulated during pathogen infection, the biological role of most of them remains unknown. Furthermore, these studies have been conducted mainly in the model dicotyledonous plant Arabidopsis thaliana during interaction with the bacterial pathogen Pseudomonas syringae. Further experimental validation is required to better understand the regulatory roles of miRNAs in plant immunity.

In the past few years, studies have demonstrated that miRNAs act as regulators of nutrient homeostasis in plants by modulating the expression of genes involved in nutrient homeostasis [17]. It has been shown that miR399 and miR395 play a fundamental role in phosphate and sulfur homeostasis in plants $[18,19]$. Plant miRNAs controlling nutrient homeostasis may also be important factors in controlling disease resistance. Unfortunately, miRNA-mediated mechanisms involved in disease resistance and nutrient homeostasis have been studied separately.

Iron $(\mathrm{Fe})$ is an essential microelement for plant growth required for essential redox reactions in metabolism. Fe is also required for photosynthesis and maintenance of chloroplast function [20]. However, excess Fe generates reactive oxygen species (ROS), which might cause oxidative damage to macromolecules (e.g. nucleic acids, lipids, proteins) and cellular structures [21-25]. During pathogen infection, Fe homeostasis must be carefully regulated as the host and pathogen compete for the available Fe. The pathogen must acquire this vital element from host tissues, whereas the host plant can deprive the invader of $\mathrm{Fe}$ as a defensive strategy. Mechanisms for maintaining $\mathrm{Fe}$ homeostasis need to be highly dynamic in the host plant to allow normal plant growth. Although distinct miRNAs have been shown to be responsive to Fe stress [26-30], how such alterations will affect Fe homeostasis and disease resistance remain to be determined.

Rice is one of the most important cereal crops in the world and the model plant for genomics research of monocotyledonous [31, 32]. Rice production is severely affected by blast disease caused by the fungal pathogen Magnaporthe oryzae [33]. miRNAs controlling traits of agronomic importance (e.g., tiller growth, early flowering, grain production) [34-36] and tolerance to abiotic stress (drought, salinity and cold stress) [37-39] have been described in rice. Evidence also supports variations in the accumulation of rice miRNAs during $M$. oryzae infection or treatment with $M$. oryzae elicitors [40-43], but the biological function of only a few of these pathogen-regulated miRNAs has been demonstrated. They include both positive regulators (miR7695, miR160, miR398, and polycistronic miR166k-
$166 \mathrm{~h}$ ) and negative regulators (miR164a, miR169 and miR319) of defense responses against the rice blast fungus $M$. oryzae [41, 42, 44-47]. Hence, to obtain a comprehensive understanding of the regulatory functions of miRNAs in the rice response to $M$. oryzae infection, intense experimental validation of miRNA functioning is mandatory.

We previously reported that the rice miR7695 is involved in blast resistance [41]. This particular miRNA targets an alternatively spliced transcript of OsNramp6 (natural resistance-associated macrophage pathogen 6), in particular the shortest transcript variant (OsNramp6.8), encoding an Fe and manganese transporter, the NRAMP6 protein [48]. Here we show that Fe accumulates at the sites of pathogen penetration (appressoria) and cells surrounding the infection sites in M.oryzae-infected rice leaves. MIR7695 activation results in blast resistance which was associated with local iron accumulation at the infection sites and superinduction of $P R$ and diterpenoid phytoalexin biosynthesis genes. Consequently, MIR7695 activation plants accumulated major rice phytoalexins in their leaves. These results provide new insights into the role of miR7695 in regulating immune responses and $\mathrm{Fe}$ signaling pathways in the rice- $M$. oryzae interaction.

\section{Results}

\section{M. oryzae infection alters Fe distribution in rice leaves}

In this work, we investigated the cellular distribution of Fe during $M$. oryzae infection by using the Perls staining. Perls reagent (potassium ferrocyanide) reacts with $\mathrm{Fe}^{3+}$ to form an insoluble pigment, or Prussian blue. Without pathogen infection, Perls staining revealed that Fe preferentially accumulated at stomata (Fig. 1a upper left panel). Upon pathogen challenge, iron staining showed a less uniform, but more widespread distribution in the stomatal areas pointing to a possible pathogen-induced iron mobilization (Fig. 1a upper right panel). Of interest, Perls staining revealed iron accumulation forming halo areas around the infection sites (Fig. 1a, lower panels).

To increase the sensitivity and definition of Fe detection in rice leaves, we used intensified Perls staining with $\mathrm{DAB} / \mathrm{H}_{2} \mathrm{O}_{2}$. This method takes advantage of the redox activity of the Prussian blue reagent. As previously observed by Perls staining, after Perls/DAB staining, strong black precipitates appeared at stomatal areas in mockinoculated leaves which showed a diffuse staining upon M. oryzae infection (Fig. 1b, upper panels). In those regions, Fe-stained granules were often visible (Fig. 1b, middle left panel). As well, in these regions, Fe accumulated with different intensities, with strong black precipitates in the center, surrounded by weaker and unevenly distributed halos of black precipitate (Fig. 1b, middle right panel). Higher magnification of these regions showed germinating spores and germ tubes forming appressoria, the sites where pathogen entry occurs (Fig. 1b, lower panel). 


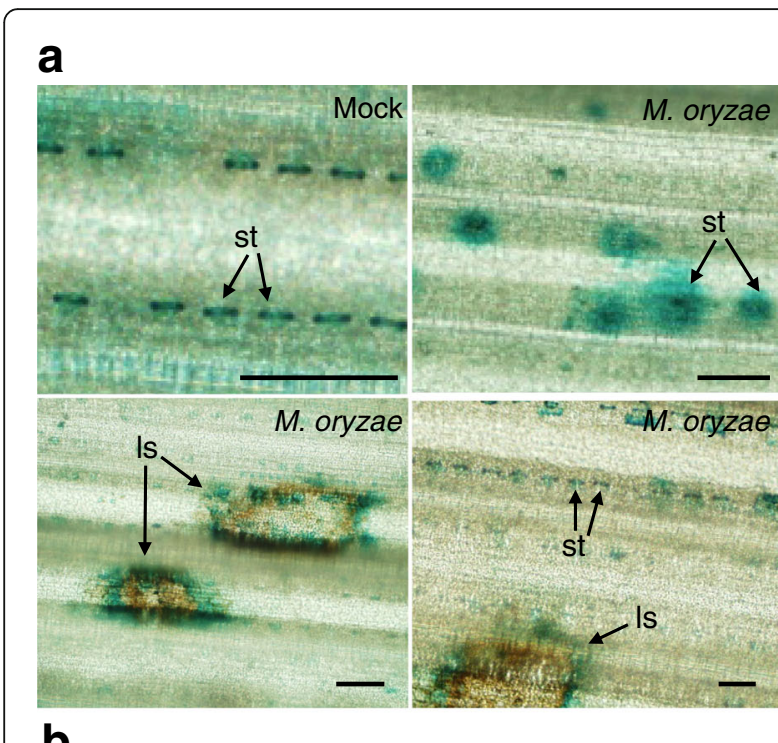

b

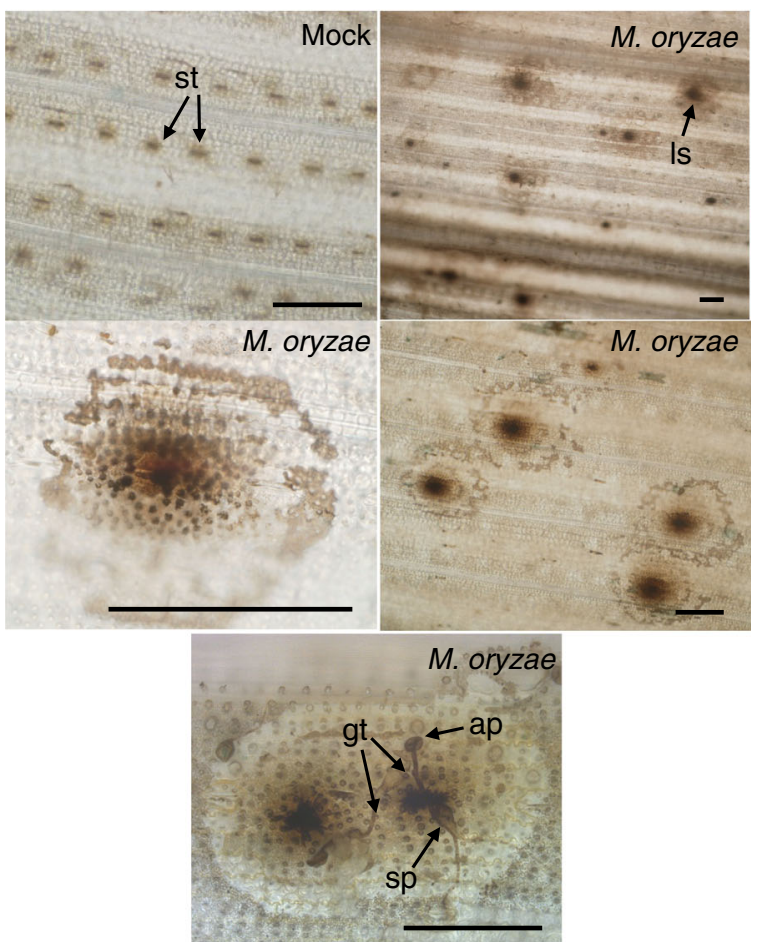

Fig. 1 Histochemical detection of iron (Fe) in M. oryzae-infected rice (O. sativa CV. Nipponbare, japonica) leaves. Wild-type plants at the three-leaf stage were sprayed with a $M$. oryzae spore suspension or mock inoculated. At 24-48 h post-inoculation (hpi), the third leaf of each plant was stained with Perls (a) or Perls/DAB (b) (blue and black precipitates, respectively). Scale bar: $100 \mu \mathrm{m}$. ap, appressorium; gt, germ tube; Is, lesion; st, stomata; sp, spore

Fe was weakly stained further away from the penetration site. Hence, histochemical analysis of Fe accumulation established that Fe accumulates at the sites of attempted penetration by the fungus (appressoria) as well as in cells in close proximity to the infection site, supporting that Fe distribution might be important for blast resistance.

\section{Resistance to infection by the rice blast fungus $M$. oryzae in mutant plants with MIR7695 activation}

We searched publicly available rice mutant collections for mutants with affected MIR7695 expression. Because of the small size of $M I R$ genes, identifying mutant alleles for miRNAs in insertional mutant collections is unlikely. A T-DNA tagged line (M0107013) was identified in the Taiwan Rice Insertion Mutants (TRIM) Database [49]; http://trim.sinica.edu.tw) in which the T-DNA was inserted upstream of the MIR7695 locus (Additional file 1: Figure S1a, left panel). TRIM was designed for gene knockout and activation tagging in the Tainung67 (japonica) background. Thus, the presence of an octamer of the cauliflower mosaic virus $35 S$ (CaMV35) transcriptional enhancer next to the left border of the T-DNA can activate the expression of genes located up to $30 \mathrm{~Kb}$ from the integration site $[50,51]$ (Additional file 1 : Figure S1a, left panel). Homozygous and azygous plants were identified by PCR genotyping (Additional file 1: Figure S1a, right panel, primers are in Additional file 2: Table S1). Quantitative PCR (qPCR) revealed that MIR7695-Ac plants had a single copy of T-DNA inserted in its genome (Additional file 3: Table S2) Importantly, the accumulation of miR7695 precursor and mature sequences was higher in homozygous mutant plants with MIR7695 activation tagging (hereafter MIR7695-Ac) than wild-type azygous (WT-Az) plants as revealed by RT-qPCR and small-RNA northern blot analyses, respectively (Fig. 2a, left panel). Consistent with upregulated MIR7695, the accumulation of miR7695 target transcripts (OsNramp6.8) was decreased in leaves with MIR7695-Ac (Fig. 2a, right panel).

We previously reported that the recognition site of miR7695 locates in the 3' UTR region of OsNramp6.8 transcripts. In this study, we further investigated whether OsNramp6.8 gene is a real target gene for miR7695 by performing RNA ligase-mediated 5' RACE (5'-RLM-RACE). Sequencing of the $5^{\prime}$-RACE PCR products identified cleavage fragments at the expected site of OsNramp6.8 transcripts, thus, supporting that OsNramp6.8 transcripts are cleaved by miR7695 (Fig. 2b). These observations demonstrated that M0107013 is an activation mutant for MIR7695 (MIR7695-Ac plants) and that miR7695 cleaves OsNramp6.8 transcripts. MIR7695-Ac plants were slightly shorter and contained less chlorophyll than did WT-Az plants, but these differences were not statistically significant (Additional file 1: Figure S1b and c).

Infection experiments were performed to assess the effect of MIR7695 activation on disease resistance. WT-Az and MIR7695-Ac plants were spray-inoculated with $M$. oryzae spores. On visual inspection, MIR7695-Ac plants 


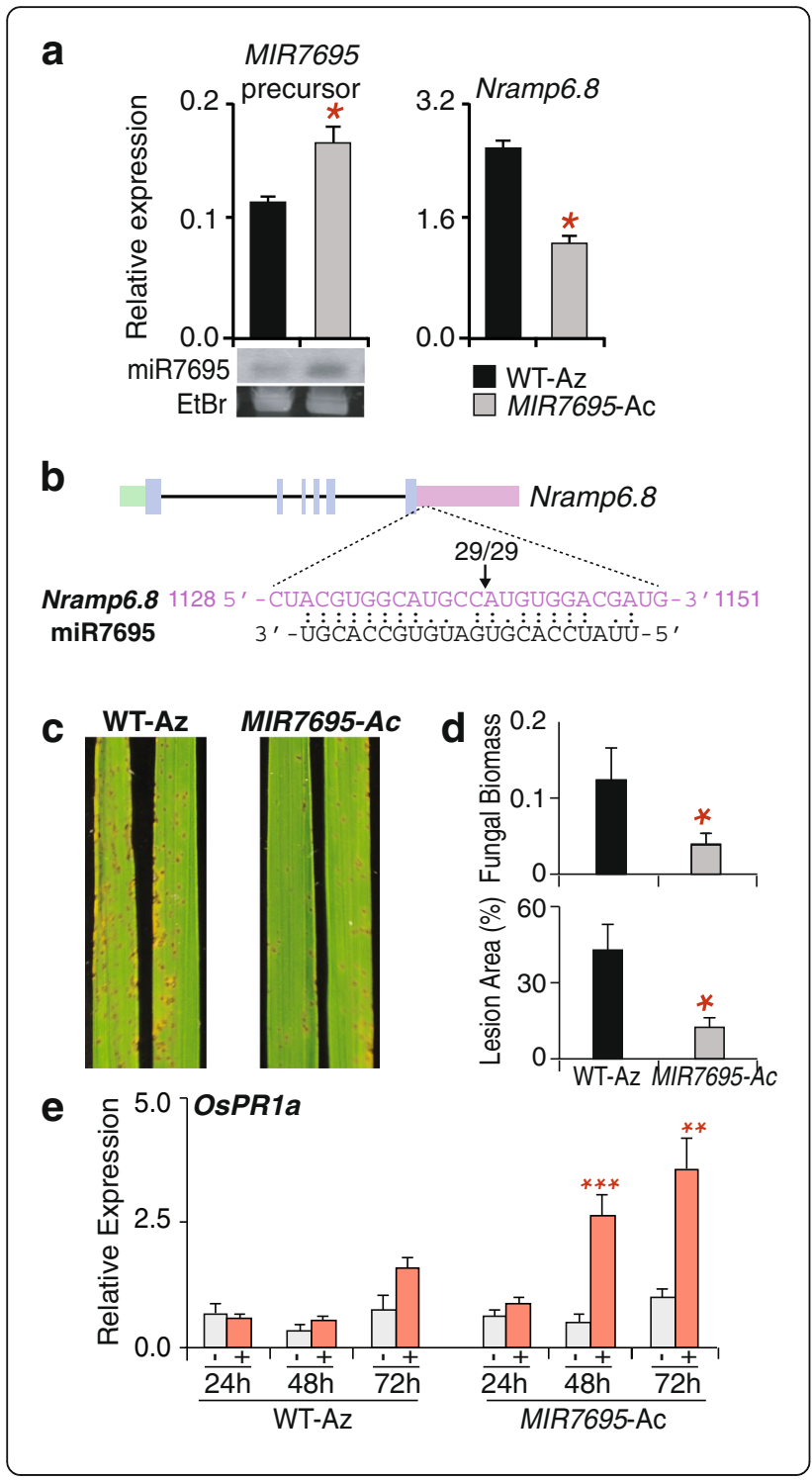

were more resistant to $M$. oryzae infection than were WT plants (Fig. 2c). Blast resistance was confirmed by quantifying the lesion area and the relative amount of fungal DNA in infected leaves (Fig. 2d). Resistance of MIR7695Ac plants to $M$. oryzae infection was also observed by local inoculation of detached rice leaves (Additional file 4: Figure S2).

The induction of $P R 1$ expression is a widely used indicator of defense activation in response to pathogen infection in plants, including infection by $M$. oryzae in rice [52]. As expected, PR1a was induced in WT-Az plants during $M$. oryzae infection (Fig. 2e). However, PR1a was induced at a much higher level in fungal-infected MIR7695-Ac than WT-Az plants (Fig. 2e), which is consistent with the phenotype of blast resistance observed in MIR7695-Ac plants. The observed phenotype of blast resistance in MIR7695-Ac plants also agreed with resistance to $M$.
Fig. 2 Resistance of MIR7695-Ac mutant plants to M. oryzae infection. a RT-qPCR analysis of MIR7695 precursor transcripts (left panel) and miR7695 target (Nramp6.8, Os01g0503400.8) in homozygous mutant (MIR7695-Ac) and WT (segregated azygous, WT-Az) plants. Data are mean \pm SE $(n=3)$ (Student $t$ test, $\left.{ }^{*} p<0.05\right)$. Lower panel: northern blot analysis of mature miR7695 using the miR7695.3-3p sequence as the hybridization probe (Additional file 2: Table S1). As a loading control, the RNA blot was stained with ethidium bromide (EtBr) (b) Experimental validation of miR7695-mediated cleavage of OsNramp6.8 transcripts by 5'-RLM-RACE. Schematic representation of the OsNramp6.8 (upper panel), showing the coding sequence (blue), $5^{\prime} U T R$ (green), and $3^{\prime} U T R$ (pink). Boxes, exons; lines, introns. Genespecific primers were used for $5^{\prime}$-RACE and the resulting PCR products were sequenced. The identified cleavage site is indicated by an arrow and the number above indicate the detected cleavage site of independent clones. c Leaves of 3-week-old plants were sprayed with a M. oryzae spore suspension. The second leaf was photographed at 7 days post-inoculation. $\mathbf{d}$ Percentage of leaf area affected by blast lesions (upper panel). Relative fungal biomass (lower panel) was determined by qPCR as the ratio of M. oryzae $28 \mathrm{~S}$ ribosomal DNA to the rice Ubiquitin1 gene (primers in Additional file 2: Table S1). Data are mean \pm SE $(n=7)$ from 1 experiment (Student $t$ test, $\left.{ }^{*} p<0.05\right)$. Four independent infection assays were performed with similar results. e RT-qPCR analysis of OsPR1a transcripts at different times after inoculation with $M$. oryzae spores. Blast infection was carried out as in (c). Data are mean \pm SE $(n=3$, each biological replicate is a pool of 3 individual leaves) (Student $t$ test, ${ }^{* *} p<0.01$ ${ }^{* * *} p<0.001$; infected vs non-infected). Mock inoculated (control) plants; + , M. oryzae-infected plants.

oryzae infection in miR7695-overexpressing lines and Osnramp6 mutant plants [41, 48].

As previously mentioned, without pathogen infection, iron accumulated in the stomata of leaves from wild-type rice plants whereas $M$. oryzae infection induced iron mobilization to the infection sites in wild-type plants (see Fig. 1). In this work, we determined the accumulation of iron at different time points after inoculation with $M$. oryzae spores in wild-type and MIR7695-Ac plants. As it was observed in wild-type plants, iron was detected in stomata of MIR7695-Ac leaves in non-infected plants (Fig. 3a). This analysis also revealed a stronger iron accumulation at the infection sites in the MIR7695-Ac plants compared to the WT-Az at 24 hpi (Fig. 3b, upper panels). Moreover, a general decrease on the iron content occurred at later time points (48 hpi, $72 \mathrm{hpi}$ ) in both wild-type and MIR7695-Ac plants.

\section{Transcript profiling of MIR7695-ac mutant plants}

To investigate the molecular mechanisms underlying blast resistance in MIR7695 plants, we used RNA-seq analysis. Initially, we examined the impact of MIR7695 activation on the rice transcriptome by comparing the transcript profiles of mock-inoculated MIR7695-Ac and WT-Az plants. We identified 281 differentially expressed genes (DEGs; 153 upregulated and 128 downregulated) (Fig. 4a; Additional file 5: Figure S3a). Additional file 6: Table S3 lists the DEGs in MIR7695-Ac plants. Singular 

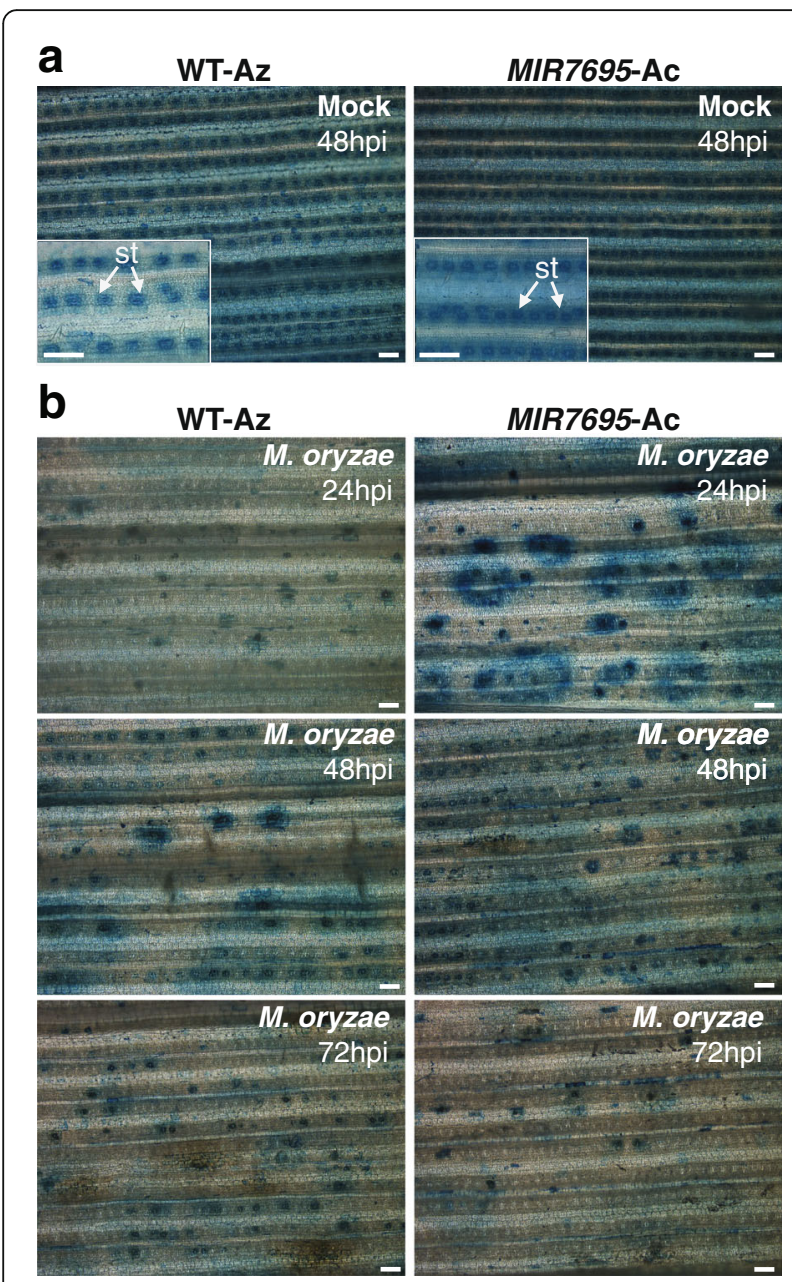

Fig. 3 Histochemical detection of iron (Fe) in wild-type (O. sativa cv. Tainung 67, japonica) and MIR7695-Ac mutant plants during $M$. oryzae infection. Wild-type and MIR7695-Ac mutant plants at the three-leaf stage were (a) mock-inoculated or (b) inoculated with $M$. oryzae spores. At 24, 48, $72 \mathrm{~h}$ post-inoculation (hpi), the third leaf of each plant was stained with Perls. Iron is detected as blue precipitates. Representative images of one experiment are shown $(n=4)$. Three independent infection assays were performed with similar results. Scale bar: $100 \mu \mathrm{m}$. st, stomata

enrichment analysis (SEA) of molecular function by using AgriGO revealed gene ontology (GO) annotations in the "binding" and "catalytic activity" categories, which were over-represented for both upregulated and downregulated DEGs (Fig. 4a; Additional file 7: Table S4). Genes in the categories "transcription regulator activity" and "transporter activity" were specifically enriched in the upregulated DEGs, whereas genes in the "electron carrier activity" category were enriched in downregulated DEGs (Fig. 4a). The binding category comprised genes related to "calcium ion binding" and "zinc ion binding" (upregulated only in MIR7695-Ac plants) and "iron ion binding" genes (downregulated only in MIR7695-Ac plants) (Fig. 4b).
The expression of a vast array of transcription factors (TFs) belonging to different TF families was regulated in mock-inoculated MIR7695-Ac plants (most of them being upregulated) (Fig. 4c; Additional file 8: Table S5). They included TFs with a demonstrated role in the rice defense response to blast infection), such as OsWRKY45 and OsNAC4 [53]. These TFs function as positive regulators of the rice response to $M$. oryzae infection $[54,55]$ and are both upregulated in MIR7695-Ac plants (Fig. 4c). Other TFs that are activated in MIR7695 plants are known to mediate defense hormone signaling, such as ethylene response factor 5 (OsEREBP5), APETALA2/ethylene-responsive element binding protein (AP2/EREBP), several jasmonate ZIM-domain (JAZ) TFs, and RERJ1 (a jasmonic acid-dependent stress inductive bHLH transcription factor) [56] (Fig. 4c). Genes encoding several wall-associated kinase (WAK) receptors and disease resistance (R) proteins were also upregulated in MIR7695-Ac (Fig. 4c). Upregulated genes in MIR7695-Ac plants also included several heavy metal transporter/metal detoxification $(H M T D)$ protein genes and siroheme uroporphyrinogen methyltransferase1 (SUM1), encoding enzymes responsible for the synthesis of the Fe-containing cofactor of enzymes (Fig. 4c).

Genes that were downregulated in mock-inoculated MIR7695-Ac plants included those involved in the synthesis of nicotinamine (NA), a chelator of metals and the precursor of phytosiderophores (components for $\mathrm{Fe}$ acquisition) [57]: OsNAS1 and OsNAS2, encoding nicotinamine synthases (Fig. 4c). Other downregulated genes are involved in oxidation-reduction processes, such as laccases (OsLAC4, OsLAC19, OsLAC24) and peroxidases (Prx81, Prx125) (Fig. 4c).

RT-qPCR was used to validate RNA-seq findings. RTqPCR results obtained for selected genes were highly concordant with RNA-seq results for both upregulated genes (OsWRKY45, OsWRKY71, OsNAC4, OsDREB1G, OsDRE $B 1 E$, OsRERJ1) and downregulated genes (OsLAC19 and OsNAS1) (Additional file 9: Figure S4).

Together, these observations suggest that without pathogen infection, MIR7695 activation led to altered expression of genes involved in 1) transcriptional regulation, 2) disease resistance, 3) metal binding and transport, and 4) oxidationreduction mechanisms. Transcriptional changes caused by MIR7695 activation might well contribute to the resistance response of these plants to pathogen infection.

\section{Enhanced defense responses to $M$. oryzae infection in MIR7695-ac plants}

Pathogen-induced alterations in the transcriptome of MIR7695-Ac plants were identified and compared to those of fungal-infected WT-Az plants. The number of genes with expression affected by $M$. oryzae infection at $48 \mathrm{~h}$ post-infection (hpi) was 4.5 times higher in MIR7695-Ac 


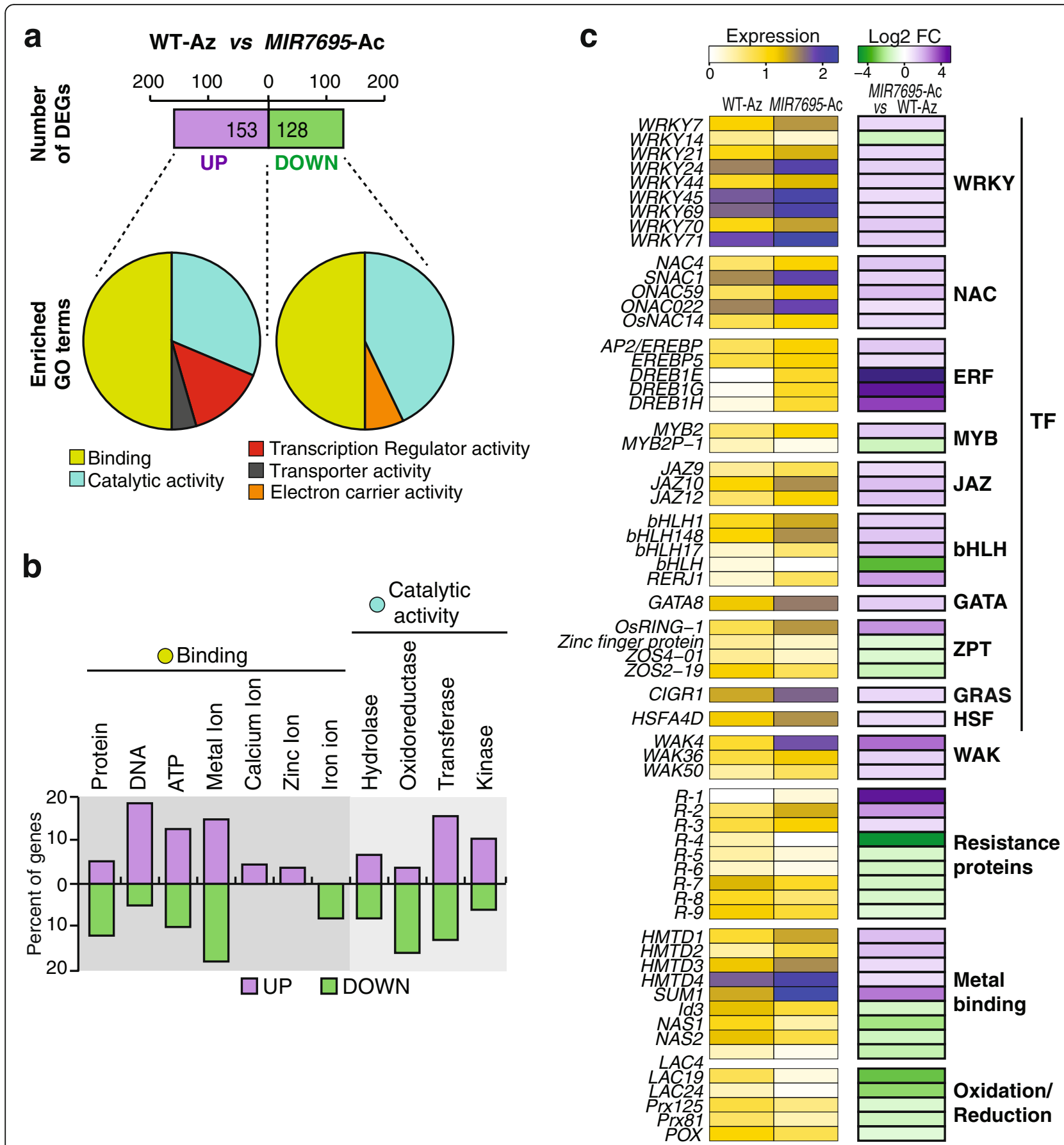

Fig. 4 Differentially expressed genes (DEGs) in MIR7695-Ac mutant plants relative to WT-Az plants by RNA-seq analysis. Leaves of three-week-old plants were used (a) Number of DEGs and Gene Ontology (GO) analysis of DEG function. Up-regulated genes (log2 fold change [FC] $\geq 1$; purple) and down-regulated genes ( $\log 2 \mathrm{FC} \leq-1$; green) genes $(p<0.05$, false discovery rate $[\mathrm{FDR}]<0.05, n=2)$. Pie charts represent the five general GO terms enriched in up- and downregulated DEGs. b Enriched terms in the "binding" and "catalytic activity" categories. c Heatmaps showing RNAseq expression level (left panel; log10 [FPKM+1]) and FC (right panel; log2FC) of DEGs. Gene expression is represented from pale yellow (less expressed) to blue (more expressed). Upregulated (log2FC $\geq 1$; purple) and downregulated (log2FC $\leq-1$; green) DEGs. Data are means $(n=2)$. The full gene names and ID list are in Additional file 8: Table S5

than WT-Az plants (531 and 116, respectively) (Fig. 5a; Additional file 5: Figure S3bc). This observation already indicated stronger transcriptional regulation in the mutant plants. DEGs for WT-Az and MIR7695-Ac plants are listed in Additional files 10 and 11 (Tables S6 and S7), respectively. Of note, genes typically associated with the plant 


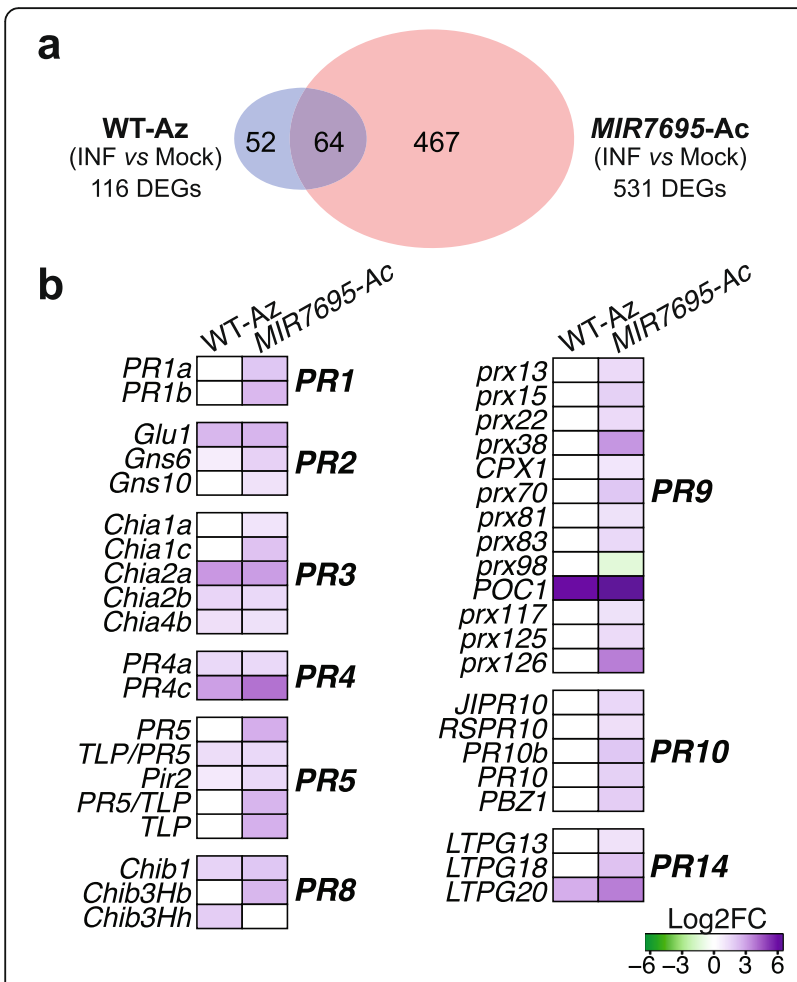

Fig. 5 Comparison of DEGs in MIR7695-Ac and WT plants after challenge with M. oryzae. Leaves of 3-week-old rice plants (WT-Az and MIR7695-AC) were mock-inoculated or sprayed with a suspension of M. oryzae spores, and collected at $48 \mathrm{hpi}$ for RNA extraction and RNA-seq. Upregulated $(\log 2 F C \geq 1)$ and downregulated $(\log 2 \mathrm{FC} \leq 1)$ genes by $M$. oryzae infection $(p<0.05$, FDR $<0.05)$. a Venn diagram of the overlap between fungalresponsive genes of each genotype. $\mathbf{b}$ Comparison of the expression pattern of defense-related genes with $M$. oryzae infection. Up(purple) and downregulated (green) DEGs. For a full list of gene IDs, see Additional file 12: Table S8

response to pathogen attack, such as $P R$ genes, were induced in MIR7695-Ac but not in WT-Az plants at 48hpi (Fig. 5b; Additional file 12: Table S8). They included PR1, $\beta$-1,3-glucanase (PR2), chitinase (PR3, PR4, PR8), thaumatin (PR5), peroxidase (PR9), PBZ1 and other Bet $v 1$ homologues (PR10), and lipid transfer protein (LTP; PR14). The antimicrobial activity of many of these PR proteins has been demonstrated (e.g., PR1, chitinases, $\beta$-1,3-glucanases, PR4, thaumatin, LTPs) [58].

To further establish differences in the transcriptional response to pathogen infection between MIR7695-Ac and WT-Az plants, we used a two-factor analysis (genotype and treatment) of the full dataset of DEGs in each genotype. A total of 153 and 100 genes were identified as upregulated and downregulated, respectively, in MIR7695-Ac versus WT plants (Fig. 6a and Additional file 16: Figure S3d; Additional file 13: Table S9). AgriGO was used for GO enrichment analysis of DEGs in the bifactorial analysis, with clustering by Revigo (Fig. 6a). The GO terms over- represented in upregulated genes were clustered in the categories "response to stress" (biotic and oxidative stress), "response to stimulus", and "secondary metabolism" (phenylpropanoids and terpenoids) (Fig. 6a, upper panel). Genes induced in MIR7695-Ac plants in the bifactorial analysis included defense-related genes, such as $P R$ genes (PR1, PR2, PR5 and PR10 family members) and oxidative stress-related enzymes (e.g., several peroxidases) (Fig. 6b and Additional file 14: Table S10).

Phenylpropanoid biosynthetic genes were highly represented in the bifactorial analysis of DEGs. They included genes involved in the production of flavonoids (CHS; F3H, FLS, DFR) and monolignols, the building blocks of lignin (CCR, CAD, COMT, PMT, LAC, PRX) (Fig. 6b and Additional file 14: Table S10; additional information on phenylpropanoid and lignin biosynthesis genes that were differentially regulated in infected MIR7695-Ac plants is in Additional file 15: Figure S5a). The expression of flavonoid biosynthesis genes is known to be induced by pathogen infection, and certain plant flavonoids exhibited antifungal activity [59]. The accumulation of lignin in secondary cell walls provides a physical barrier against pathogen invasion [60]. The expression of several peroxidases was upregulated in MIR7695-Ac versus WT-Az plants and also with pathogen infection. Peroxidases are key enzymes in the biosynthesis of lignin during resistance reactions via cross-linking of lignin monomers. A stronger induction of genes involved in flavonoid and lignin biosynthesis might play a role in protecting the MIR7695-Ac plants against $M$. oryzae infection. In addition, a important number of genes involved in the production of diterpenoid phytoalexins were among the top induced genes in MIR7695Ac plants with infection (bifactorial DEGs) (Fig. 6b; Additional file 14: Table S10) as described below.

The GO term most represented in downregulated genes on bifactorial analysis of DEGs was "iron ion transport" (Fig. 6a, lower panel). This included genes related to $\mathrm{Fe}$ homeostasis, such OsFRO2 (a Fe ${ }^{3+}$ reductase), OsNAS2 (a nicotianamine synthase), OsIRO2 TF, OsHRZ1, OsHRZ2 ubiquitin ligases, OsNRAMP1 $\left(\mathrm{Fe}^{2+}\right.$ transporter), OsMIR (mitochondrial Fe-regulated gene), and OsIROPT (an oligopeptide transporter) (Fig. 6b; Additional file 14: Table S10).

A more detailed expression analysis was performed for genes identified by bifactorial analysis, and their expression was examined by RT-qPCR at different times after inoculation with $M$. oryzae spores (24, 48 and $72 \mathrm{hpi}$ ). This analysis confirmed stronger induction of $P R$ genes (OsPR1b, OsPBZ, OsPR10b) and lignin biosynthesis genes (OsCAD2, OsCOMT1) in MIR7695-Ac than WTAz plants during $M$. oryzae infection (Fig. 7). Induction of OsPBZ1 and other OsPR 10 family members is known to occur during $M$. oryzae infection and, when overexpressed, the genes confer pathogen resistance [61-63]. 


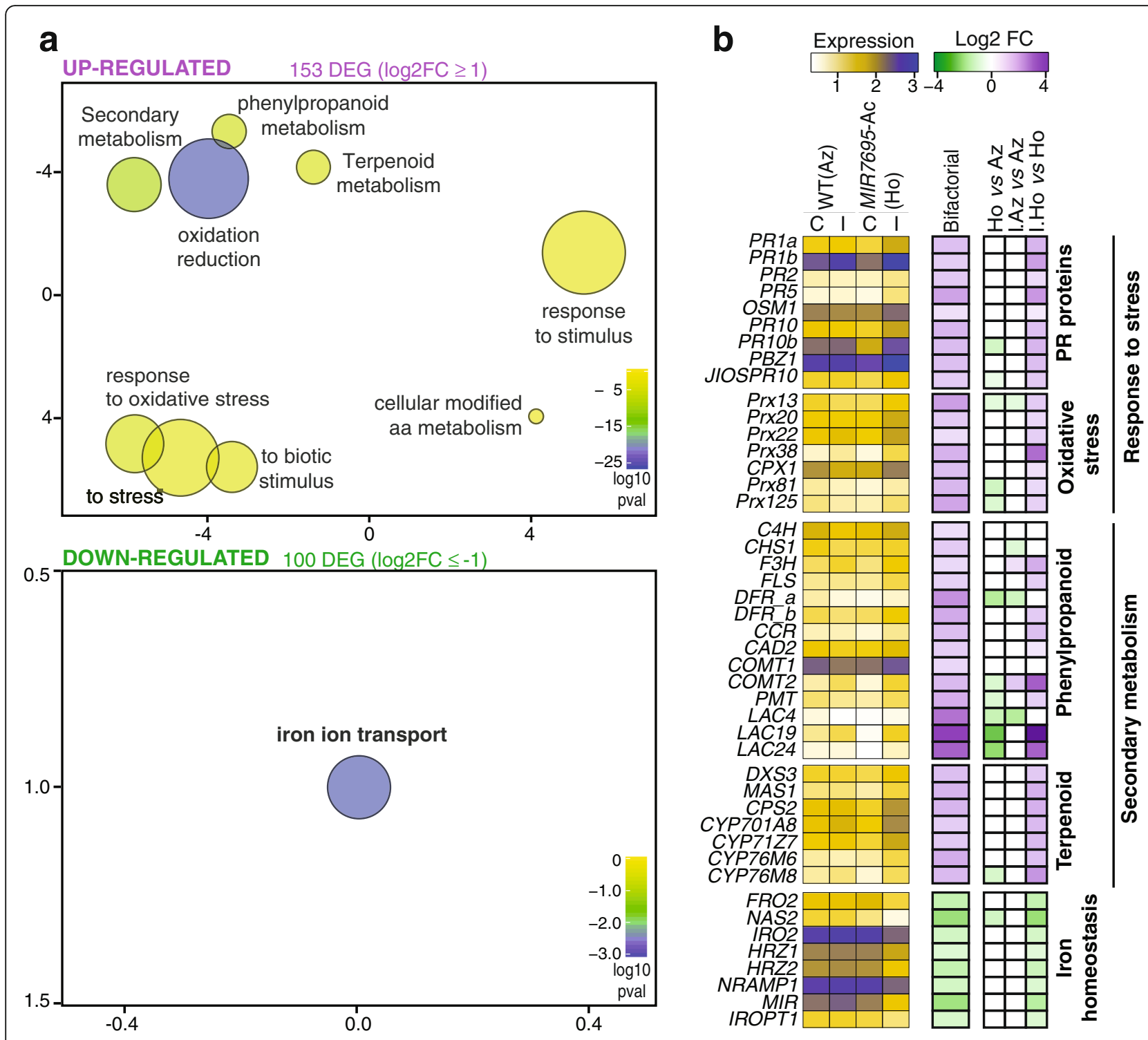

Fig. 6 Biological processes altered in MIR7695-Ac mutant plants relative to WT-Az plants with M. oryzae infection. Same plant material as in Fig. 4. RNA-seq data underwent bifactorial analysis (upregulated, $\log 2 \mathrm{FC} \geq 1$; downregulated, $\log 2 \mathrm{FC} \leq 1 ; p<0.05, \mathrm{FDR}<0.05$ ). a GO analysis of DEG function in MIR7695-Ac plants with blast infection (upper and lower panels show upregulated and downregulated DEGs, respectively). The top GO terms enriched in MIR7695-Ac vs WT-Az DEGs were represented by using REVIGO after reducing redundancy (http://revigo.irb.hr/). Circles represent GO terms and those clustered closer to each other represented similar GO terms. Disc colors (blue to yellow) represent de degree of $\mathrm{GO}$ enrichment ( $p$-value) and disc size is proportional to the frequency of the GO term in the GO database (larger and smaller discs represent more general and more specific terms, respectively). b Heatmap showing distribution of RNAseq expression level (log10[FPKM+ 1], pale yellow to blue from less to more expressed) for DEGs belonging to the top enriched GO categories in M. oryzae-infected MIR7695-Ac plants (left panel). Heatmaps show upregulated (purple) and downregulated (green) DEGs (bifactorial analysis, middle panel; Monofactorial analysis for the given comparisons, right panel) Biological processes are indicated to the left. Data are means $(n=2)$. The full gene ID list is shown in Additional file 14 : Table S10.

Altogether, comparative transcriptome analysis (bifactorial analysis) revealed stronger induction of defenserelated genes in MIR7695-Ac (e.g., PR, oxidative stressrelated, phenylpropanoid and diterpenoid phytoalexin biosynthesis genes), whereas genes that function in $\mathrm{Fe}$ homeostasis appear to be downregulated in MIR7695-Ac plants during $M$. oryzae infection.
Regulation of Fe homeostasis-related genes in rice leaves during $M$. oryzae infection

In plant roots, two different mechanisms have been described for Fe uptake from the rhizosphere, the reducing and chelating strategies (strategies I and II, respectively) $[64,65]$. Rice is unique in that as it uses both strategies. Besides strategy I and II genes, other genes contribute to 

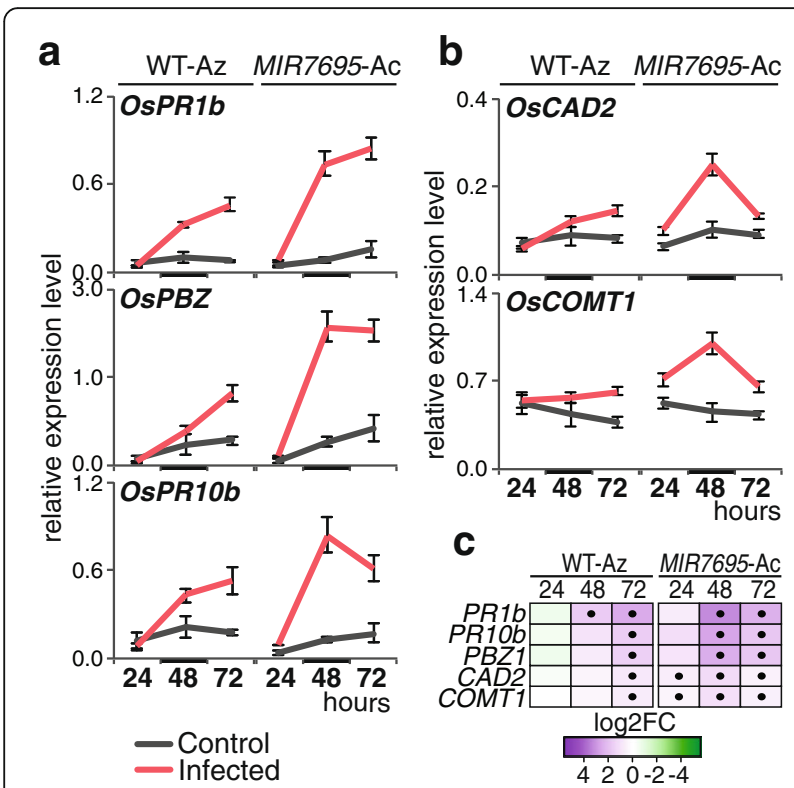

Fig. 7 Expression of $P R$ and lignin biosynthesis genes in WT-Az and MIR7695-Ac plants during blast infection. Plants were sprayed with a M. oryzae spore suspension. Leaves were collected at the indicated times (24, 48, 72 hpi). a-b Expression analysis of (a) PR (OsPR1b, OsPBZ, OsPR10b) and (b) lignin (OsCAD2, OsCOMT1) genes determined by RT-qPCR. Data are mean \pm SE $(n=3$; each sample consisted of a pool of 3 individual leaves). Mock-inoculated (control, grey) and M. oryzae-infected (red) plants. Time point used for RNAseq analysis ( $48 \mathrm{~h}$ ) is labeled with a thick line in the $\mathrm{x}$-axis. c Heatmap showing log2 FC for each transcript and each time (infected vs. control) as determined from RT-qPCR values (a-b). Upregulated (purple) and downregulated (green). Dots indicate significant differences (infected vs control) (Student $t$ test, $p<0.05$ )

Fe transport and/or mobilization through the plant. Although great progress has been made during the last years to identify mechanisms governing Fe uptake in roots, the regulation of Fe homeostasis genes in leaves is less understood.

We investigated the expression profile of Fe homeostasisrelated genes in leaves of wild-type plants during $M$. oryzae infection. Genes examined were: OsFRO2, OsIRO2, OsHRZ1, OSNRAMP1 and OsIROPT1 (genes strongly downregulated in MIR7695-Ac plants). These genes were strongly upregulated early during infection (24-48 hpi) but downregulated at a later stage of the infection process (72 hpi) (Additional file 16: Figrue S6; WT-Az, infected vs mock). Upregulation of these genes early during infection of WT plants correlates with Fe accumulation at the sites of fungal penetration and infection sites, as revealed by histochemical analysis of $M$. oryzae-infected rice leaves (Fig. 1).

For a comparison, we examined the expression profile of Fe homeostasis genes in MIR7695-Ac plants. Four of the five genes examined were induced early during infection (24 hpi), as it was observed in WT plants, followed by a strong downregulation at 48 and $72 \mathrm{hpi}$ (Additional file 16: Figure S6; MIR7695-Ac, infected vs mock). Therefore, downregulation of $\mathrm{Fe}$ homeostasis genes occurs earlier in MIR7695-Ac than WT-Az plants.

\section{Phytoalexins accumulate in MIR7695-ac plants during $M$. oryzae infection}

Phytoalexins are low-molecular-weight antimicrobial compounds that accumulate in plant tissues during pathogen infection [66]. Major phytoalexins accumulating in rice leaves in response to $M$. oryzae infection are the diterpene phytoalexins momilactones, phytocasssenes and oryzalexins [67]. As previously mentioned, the expression of genes involved in the biosynthesis of diterpenoid phytoalexins, oryzalexins, phytocassenes and momilactones was induced to a higher level in MIR7695-Ac than WT-Az plants (at 48 hpi with $M$. oryzae) (see Fig. 6b). For details on genes involved in diterpene phytoalexin biosynthesis with overexpression in MIR7695 plants, see Additional file 15: Figure S5b. RT-qPCR analysis of diterpene phytoalexin biosynthesis genes at different times after inoculation with $M$. oryzae spores (24, 48, $72 \mathrm{hpi}$ ) confirmed earlier and stronger induction of these genes in MIR7695-Ac than WT-Az plants (Fig. 8a, b). Differences in pathogen-induced expression of these genes were more evident at 48 and $72 \mathrm{~h}$ after blast inoculation, as revealed by the higher fold change of gene expression (Fig. 8c).

To investigate whether superinduction of diterpenoid phytoalexin biosynthesis genes affects phytoalexin accumulation, we measured phytocassane and momilactone levels in leaves of MIR7695-Ac and WT-Az plants without and with infection. As expected, the expression of phytocassanes (B, C and E) and momilactones (A and B) was barely detected in non-infected rice leaves (Fig. 8d). Upon pathogen challenge, the accumulation of phytocassanes and momilactones increased in both WT-Az and MIR7695-Ac plants but was significantly higher in MIR7695-Ac than WT plants (Fig. 8d). These findings revealed that in response to pathogen infection, diterpenoid phytoalexin biosynthesis genes are induced earlier in MIR7695-Ac than WT-Az plants. MIR7695-Ac plants also accumulated higher levels of phytoalexins during pathogen infection. Knowing that diterpene phytoalexins have antifungal activity against $M$. oryzae [67-69], a higher $M$. oryzae-induced accumulation of phytoalexins in leaves of MIR7695-Ac plants might contribute to disease resistance in these plants.

Finally, we investigated whether Fe supply affects the expression of genes involved in the biosynthesis of diterpenoid phytoalexins in rice. We analyzed the effect of $\mathrm{Fe}$ supply (low, sufficient and high) on the expression of OsCPS2 and OsCPS4, which function first cyclization steps in the phytoalexin biosynthetic pathway (Additional file 15: Figure S5b). The expression of these genes was barely 


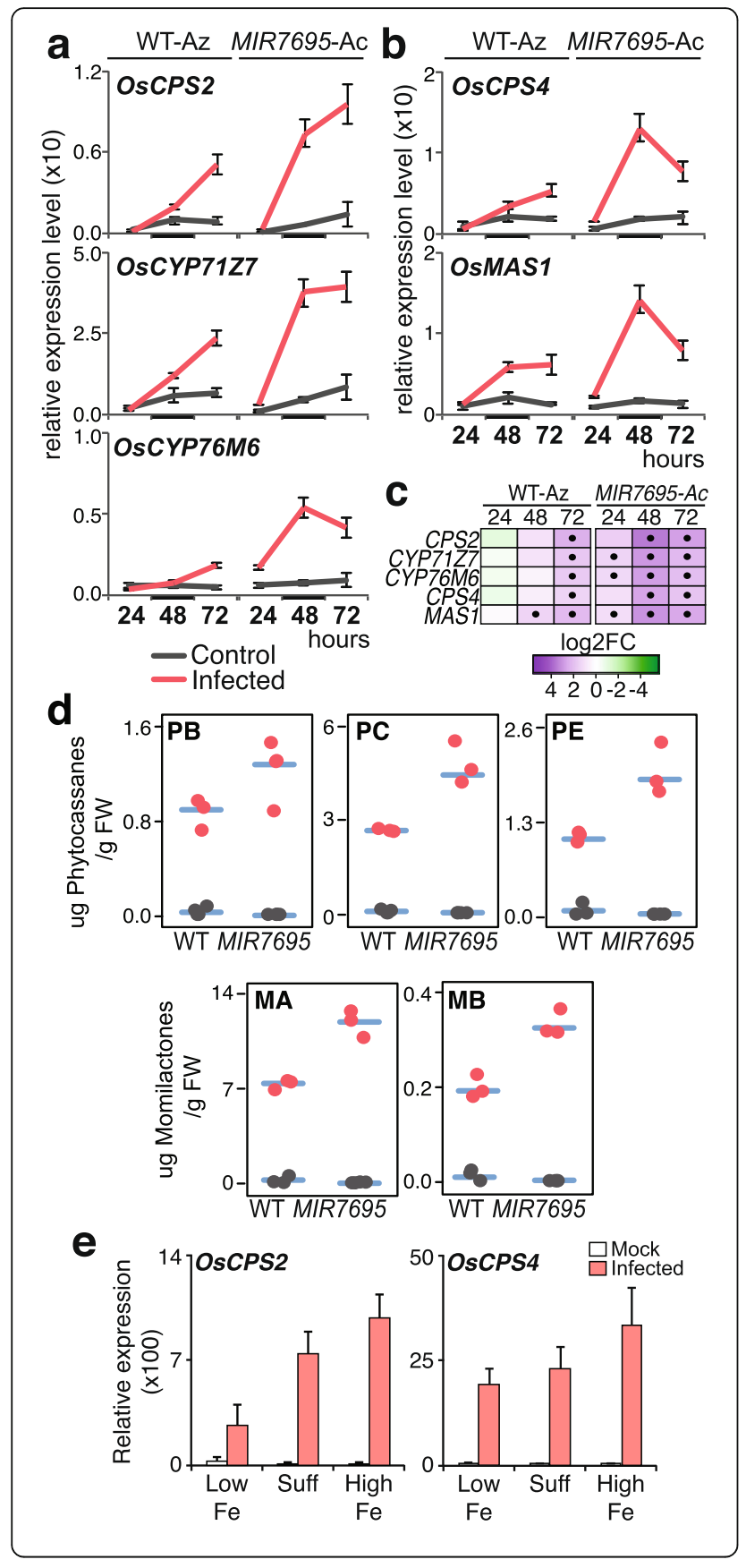

detected in plants without infection (Fig. 8e). Upon pathogen challenge, the highest expression of phytoalexin genes occurred in plants grown under high Fe supply as compared with low or sufficient Fe (Fig. 8e), which supports that Fe supply affects phytoalexin biosynthesis. Presumably, a localized accumulation of Fe at the sites of pathogen penetration and/or invasion would activate the expression of phytoalexin biosynthetic genes for phytoalexin accumulation, thus arresting fungal colonization in infected leaves of MIR7695-Ac plants.
Fig. 8 Expression of diterpenoid phytoalexin genes in WT-Az and MIR7695-Ac plants during blast infection. Plant material was treated as in Fig. 7. a-b RT-qPCR analysis of expression of (a) Phytocassane (OsCPS2, OsCYP71Z7, OsCYP76M6) and (b) momilactone (OsCPS4, OsMAS1) biosynthesis genes in rice leaves infected with M. oryzae. Data are mean \pm SE ( $n=3$; each sample consisted of a pool of 3 individual leaves). Mock-inoculated (control, grey) and M. oryzaeinfected (red) plants. c Heatmap showing log2 FC for each transcript and each time point (infected vs control) as determined from RTqPCR values (a-b). Upregulated (purple) and downregulated (green). Dots indicate significant differences (infected vs control) (Student $t$ test, $p<0.05)$. d Accumulation of diterpenoid phytoalexins, phytocassane $E(P E), B(P B)$ and $C(P C)$ (upper panels) and momilactone $A(M A)$ and $B(M B)$ (lower panels), in leaves of mockand $M$. oryzae-infected plants. Each dot represents a biological replicate. FW, fresh weight. e RT-qPCR of expression of upstream diterpenoid biosynthetic genes (OSCPS2 and OsCPS4) in mock- and M. oryzae-infected leaves of rice plants treated under three different Fe supply conditions (low, sufficient, high). Data are mean \pm SE ( $n=$ 3 ), each sample consisting of a pool of 4 individual leaves)

\section{Discussion}

Although an increasing number of miRNAs have been shown to be differentially expressed in response to pathogen infection or nutrient stress, most of this research involved plants exposed to one or another type of stress separately. Furthermore, few studies aimed to understand the regulation of Fe homeostasis in rice during $M$. oryzae infection. Here, we present evidence of a miR7695-guided cleavage of OsNramp6.8 transcripts encoding the NRAMP6 iron transporter from rice. Moreover, we investigated the role of miR7695 in the rice response to infection by $M$. oryzae. Upon challenge with $M$. oryzae, $\mathrm{Fe}$ accumulated near $M$. oryzae appressoria and in cells surrounding infected regions of rice leaf. Very recently, Dangol et al. reported that incompatible rice/M.oryzae interactions trigger iron- and ROSdependent ferroptotic cell death in leaf sheaths of rice plants where iron accumulated at sites of infection to mediate the oxidative burst [22].. Activation-tagged MIR7695 rice plants showed enhanced resistance and a stronger accumulation of iron at the sites of infection. On RNA-seq analysis, defense-related genes, including $P R$ and diterpenoid biosynthetic genes were strongly induced along with blast resistance in MIR7695-Ac plants. Levels of phytoalexins during pathogen infection were higher in MIR7695-Ac than WT azygous plants and genes in the phytoalexin biosynthetic pathway were highly induced in rice plants grown under high Fe supply. This piece of evidence support that miR7695 positively regulates immune responses and establish links between defense signaling and $\mathrm{Fe}$ homeostasis in rice. However, the exact mechanisms by which Fe signaling regulates the expression of defense-related genes remains to be determined. 
Being a foliar pathogen, $M$. oryzae has an absolute requirement for Fe from host tissues, so rice plants might capitalize on the toxicity or the essentiality of $\mathrm{Fe}$ to arrest $M$. oryzae invasion. Different scenarios can be considered. On the one hand, mechanisms that exploit Fe toxicity might be used by the host plant against $M$. oryzae. On the other, the host plant might develop withholding strategies to restrict Fe availability to the invading pathogen, a process that in humans and animals has been called "nutritional immunity" [70]. An examination of Fe distribution in $M$. oryzae-infected WT rice leaves revealed $\mathrm{Fe}$ accumulation in close vicinity of appressoria and in cells surrounding the infection sites, thus, reinforcing the notion that rice plants use strategies to locally increase Fe levels to prevent penetration and spread of the pathogen into the leaf tissue. Local accumulation of Fe would avoid Fe poisoning caused by a generalized accumulation of $\mathrm{Fe}$ in rice leaf while providing a signal for the activation of host immune responses. If so, this localized accumulation at the sites of pathogen penetration and invasion might mediate a localized oxidative burst that can be toxic to the invading pathogen. Local accumulation of $\mathrm{H}_{2} \mathrm{O}_{2}$ would also serve for cell-wall reinforcement (lignification, oxidative cross-linking of cell wall components) and induction of defense-related genes (e.g., $P R$ genes).

Without infection, Fe preferentially accumulated in leaf stomata. In this respect, Fe has been shown to be important in regulating aperture of stomata [71]. During $M$. oryzae infection, a re-distribution of $\mathrm{Fe}$ appears to occur in the rice leaf, Fe moving around stomata and toward the sites of pathogen penetration and colonization. In support of this notion, a localized accumulation of $\mathrm{Fe}$ in cell wall appositions and subsequent defensive $\mathrm{H}_{2} \mathrm{O}_{2}$ production was previously linked to basal defense in wheat leaves after infection with Blumeria graminis f. sp. tritici [72]. Also, altered Fe distribution in Arabidopsis plants infected with the bacterial pathogen Dickeya dadantii was reported [73]. Although not proven, the activation of toxic oxidative bursts caused by localized accumulation of Fe in rice leaves might be important to restrict $M$. oryzae growth while maintaining normal plant development. Iron accumulation at the sites of pathogen infection was observed in both wild-type and MIR7695-Ac plants, the later ones accumulating more iron at the infection sites than wild-type plants.

During $M$. oryzae infection, genes involved in $\mathrm{Fe}$ homeostasis were strongly downregulated in leaves of MIR7695-Ac plants compared with WT plants. As previously mentioned, the rice plant uses a combined strategy for Fe uptake from the rhizosphere that has features of both strategy I (reduction of $\mathrm{Fe}^{3+}$ to $\mathrm{Fe}^{2+}$, a system that operates in roots of most non-graminaceous species) and strategy II (release of phytosiderophores by the root, typical of graminaceous species) [74]. Our results indicate that during $M$. oryzae infection, genes that function in Fe uptake via strategy I (e.g. OsFRO2) or strategy II (e.g. OsIRO2, OsNAS2) in roots are downregulated in leaves in both WT-Az and MIR7695-Ac plants. Other Fe homeostasis genes such as NRAMP1 (a Fe transporter), OsHRZ1 and OsIROPT1 are also downregulated during infection. In line with this, the Fe homeostasis genes TmFER1 and TmNAS1 (marker genes for monitoring intracellular Fe status in wheat) were found downregulated in B. graminis-infected wheat leaves [72]. Furthermore, downregulation of $\mathrm{Fe}$ homeostasis genes was accompanied by cytosolic Fe depletion and induction of $P R$ genes. A better understanding of the mechanisms involved in Fe homeostasis in rice leaf tissues is needed to know whether $M$. oryzae also provokes intracellular $\mathrm{Fe}$ depletion in rice leaves.

MIR7695-Ac plants exhibited resistance to M. oryzae infection, which is consistent with the phenotype of disease resistance observed in loss-of-function OsNramp6 plants [48]. Disease resistance in MIR7695-Ac plants is associated with a basal expression of resistance genes and defense regulatory genes (e.g., OsWRKY45, OsNAC4) without pathogen infection and a superinduction of defense-related genes with infection. Thus, MIR7695-Ac plants mount a stronger defense response to pathogen infection, a response that is reminiscent of defense priming [75]. Whether defense responses are activated earlier in MIR7695-Ac than WT plants is unknown. Furthermore, proteins encoded by defense-related genes that are strongly induced during infection in MIR7695-Ac plants are known to possess antimicrobial activity (e.g., chitinases, $\beta$-1,3-glucanases, PR10 and LTP proteins), and their overexpression in plants confers pathogen resistance, including blast resistance $[63,76]$. Stronger expression and induction of peroxidases is also a feature of MIR7695-Ac plants, these genes being typically induced in host plant tissues upon pathogen infection. Peroxidases are important for generating highly toxic environments by producing ROS species during resistance reactions [77] and for lignin biosynthesis (cross-linking of lignin monomers). A miR7695-mediated regulation of peroxidases might then function to generate an oxidative burst at the sites where Fe accumulates, thus helping to limit pathogen spread on the rice leaf. Also, an important number of genes involved in the flavonoid and lignin branches of the general phenylpropanoid pathway were upregulated in MIR7695$A c$ versus WT plants (bifactorial analysis). The antifungal activity of phenylpropanoid compounds against phytopathogens has been reported [78-80]. The superactivation of these various defense genes might be responsible for the blast resistance phenotype observed in MIR7695-Ac plants.

Notably, upon pathogen challenge, diterpenoid phytoalexin biosynthesis genes were highly upregulated in MIR7695-Ac plants (bifactorial analysis), accompanied 
by increased accumulation of major rice phytoalexins. For some of these phytoalexins, antifungal activity against $M$. oryzae has been described [67-69]. Other studies proposed that rapid biosynthesis of diterpene phytoalexins contributes to resistance to $M$. oryzae, whereas delayed induction of these genes results in enhanced susceptibility to blast infection [67]. The accumulation of phytoalexins would enhance the ability to cope with pathogen infection in MIR7695-Ac plants.

\section{Conclusions}

Overall, this study highlights the relevance of miR7695 in blast resistance via regulation of rice immune responses. Because miR7695 regulates OsNramp6 encoding a Fe transporter from rice, these results support the existence of links between miR7695/OsNramp6 functioning to control Fe signaling and defense signaling in rice. At the cellular level, $M$. oryzae infection altered Fe distribution in rice leaves, a process probably involving miR7695. Because miRNAs function as fine-tuners of gene expression instead of turning-on or turning-off target gene expression, miR7695 would be well suited to maintain appropriate Fe levels in host cells during pathogen infection. If so, miR7695 might well be involved in modulation of iron accumulation in tissues of the rice leaf which, in turn, would affect the expression of Fe homeostasis genes. The current challenge of basic and applied plant research is to understand interconnected regulations between miR7695mediated mechanisms involved in Fe homeostasis and disease resistance in plants. Deciphering the mechanisms involved in Fe distribution and remobilization during $M$. oryzae infection with the participation of miR7695 will help in designing innovative strategies for blast disease control. Knowing how plants integrate immune responses and Fe signaling pathways is an issue of great importance in both basic and applied plant research.

\section{Methods}

Plant material, growth conditions and genotyping

Rice plants were grown at $28{ }^{\circ} \mathrm{C}$ with a $14 \mathrm{~h} / 10 \mathrm{~h}$ light/ dark cycle. The T-DNA insertion line for MIR7695 (O. sativa cv. Tainung67, japonica) was obtained from the Taiwan Rice Insertion Mutant (TRIM) collection from the Academia Sinica of Taiwan ([49]; http://trim.sinica. edu.tw), and propagated under controlled conditions (CRAG greenhouse Service). For genotyping, genomic DNA was extracted as described [81] but with mixed alkyltri-methylammoniumbromide (MATAB) used as the extraction buffer $(0.1 \mathrm{M}$ Tris- $\mathrm{HCl} \mathrm{pH} 8.0,1.4 \mathrm{M}$ $\mathrm{NaCl}, 20 \mathrm{~mm}$ EDTA, 2\% MATAB, 1\% PEG 6000, 0.5\% sodium sulphite). PCR genotyping (100 ng DNA/PCR reaction) involved specific primers (P1 and $P 3)$ and $\mathrm{T}$ DNA-specific primers (P2) (Additional file 2: Table S1). T-DNA copy number was estimated as described [82].
For Fe treatment, 10 rice seeds were grown in $0.35-\mathrm{L}$ pots containing soil (turface: vermiculite:quartz sand [2:1: 3]) for 14 days and then watered with a half-strength Hoagland solution $\left(5 \mathrm{mM} \mathrm{KNO} 3,5 \mathrm{mM} \mathrm{Ca}\left(\mathrm{NO}_{3}\right)_{2} \cdot 4 \mathrm{H}_{2} \mathrm{O}, 2\right.$ $\mathrm{mM} \mathrm{MgSO}_{4} \cdot 7 \mathrm{H}_{2} \mathrm{O}, 1 \mathrm{mM} \mathrm{NH} \mathrm{NO}_{3}, 0.5 \mathrm{mM} \mathrm{KH}_{2} \mathrm{PO}_{4}(\mathrm{pH}$ to 6.0), $46.3 \mu \mathrm{M} \mathrm{H} \mathrm{H}_{3} \mathrm{BO}_{3}, 9.1 \mu \mathrm{M} \mathrm{MnCl} \mathrm{Mn}_{2} \cdot 4 \mathrm{H}_{2} \mathrm{O}, 0.76 \mu \mathrm{M}$ $\mathrm{ZnSO}_{4} \cdot 7 \mathrm{H}_{2} \mathrm{O}, \quad 0.2 \mu \mathrm{M} \quad \mathrm{CuSO}_{4} \cdot 5 \mathrm{H}_{2} \mathrm{O}, \quad 0.28 \mu \mathrm{M}$ $\mathrm{Na}_{2} \mathrm{MoO}_{4} \cdot 2 \mathrm{H}_{2} \mathrm{O}, 51.7 \mu \mathrm{M}$ Fe-EDDHA). To assess the effect of Fe supply, the same nutrient solution was used but with a lower or higher Fe concentration $(0.1 \mu \mathrm{M}$ or $1 \mathrm{mM}$ Fe-EDDHA). After 5 days of Fe treatment, plants were infected with $M$. oryzae spores (see below for inoculation method).

\section{Perls staining and $\mathrm{DAB} / \mathrm{H}_{2} \mathrm{O}_{2}$ intensification}

Rice leaves (mock- and blast-inoculated, $48 \mathrm{~h}$ postinfection [hpi]) were stained with Prussian blue dye according to [83] with some modifications. Briefly, rice leaves were vacuum-infiltrated in a fixing solution (chloroform:methanol:glacial acetic acid; 6:3:1, v/v) for $1 \mathrm{~h}$ and incubated overnight at room temperature. After washing with distilled water (three times), samples were vacuuminfiltrated with a pre-warmed $\left(37^{\circ} \mathrm{C}\right)$ staining solution $(4 \%$ $\mathrm{HCl}$ and $4 \% \mathrm{~K}$-ferrocyanide at equal volumes) for $1 \mathrm{~h}$, incubated $1 \mathrm{~h}$ more at $37^{\circ} \mathrm{C}$ in the same solution without vaccuum and washed three times with distilled water (Perls staining). For DAB intensification reaction, samples were incubated in a methanol solution $(0.01 \mathrm{M} \mathrm{NaN}, 0.3 \%$ [v/v] $\mathrm{H}_{2} \mathrm{O}$ ) for $1 \mathrm{~h}$, washed with $0.1 \mathrm{M}$ phosphate buffer $\mathrm{pH} 7.2$, then incubated with the intensification solution (0.025\% [w/v] DAB [Sigma], 0.005\% [v/v] $\mathrm{H}_{2} \mathrm{O}$ in $0.1 \mathrm{M}$ phosphate buffer, $\mathrm{pH} 7.2$ ) for $15 \mathrm{~min}$. The reaction was stopped by washing with distilled water. Leaves were mounted in glycerol 50\% in glass slides and observed under a microscope (AixoPhot DP70 under with light).

\section{Chlorophyll content}

The mean of 10 readings from the chlorophyll meter (SPAD 502 Plus Chlorophyll Meter, Spectrum Technologies) was obtained from the third leaf of rice plants grown in different Fe concentrations. The measurement was taken at the same position in all leaves.

\section{Blast resistance assays}

The fungus M. oryzae (strain Guy-11, courtesy of Ane Sema) was grown in Complete Media Agar (CMA, $9 \mathrm{~cm}$ plates, containing $30 \mathrm{mg} / \mathrm{L}$ chloramphenicol) for 15 days at $28{ }^{\circ} \mathrm{C}$ under a $16 \mathrm{~h} / 8 \mathrm{~h}$ light/dark photoperiod condition. $M$. oryzae spores were prepared as previously described [41]. Soil-grown plants (3-4 leaf stage) were infected by two different methods, 1) whole-plant spray inoculation assays [84], and 2) drop inoculation on detached leaves [85]. Briefly, the spray inoculation method consisted of spraying whole rice plants with a $M$. oryzae 
spore suspension $\left(10^{5}\right.$ spores $/ \mathrm{ml} ; 0.2 \mathrm{ml} /$ plant $)$ by using an aerograph at $2 \mathrm{~atm}$ of pressure. Plants were maintained overnight in the dark under high humidity. For the drop inoculation method, the second detached leaf was placed into square plate dishes (12 leaves/plate) with $1 \%(\mathrm{w} / \mathrm{v})$ water agar containing kinetin $(2 \mathrm{mg} / \mathrm{l})$. Then, Whatman filter paper discs saturated with a $M$. oryzae spore suspension $\left(10^{4}-10^{6}\right.$ spores $\left./ \mathrm{ml}\right)$ were placed onto the upper face of the leaf for $60 \mathrm{~h}$. The percentage of leaf area affected by blast lesions was determined at 4 days (drop-inoculated leaves) or 7 days (spray-inoculated leaves) post-inoculation with $M$. oryzae spores by using the APS Assess 2.0 program [86].

\section{Expression analysis}

Total RNA was extracted from plant tissues by using TRizol reagent (Invitrogen). For northern blot analysis of rice miRNAs, RNAs were fractionated in a $17.5 \%$ denaturing polyacrylamide gel containing $8 \mathrm{M}$ urea, transferred to nylon membranes and probed with a $\gamma{ }^{32} \mathrm{P}$-ATP endlabeled miR7695.3-3p oligonucleotide (Additional file 2: Table S1). Blots were pre-hybridized and hybridized in Perfect-Hyb Plus buffer (Sigma) at $42^{\circ} \mathrm{C}$. Hybridization signals were detected by using STORM Phosphorimager (GE Healthcare).

For quantitative RT-PCR (RT-qPCR), the first complementary DNA was synthesized from DNase-treated total RNA $(1 \mu \mathrm{g})$ with High Capacity cDNA Reverse Transcription (Life technology, Applied Biosystems). Amplification involved $2 \mu \mathrm{l} \mathrm{cDNA}(5 \mathrm{ng} / \mu \mathrm{l})$ in optical 96-well plates (Roche Light Cycler 480; Roche Diagnostics, Mannheim, Germany) with SYBR Green I dye and gene-specific primers (Additional file 2: Table S1). The Ubiquitin1 gene (Os06g0681400) was used to normalize transcript levels.

\section{5'-RLM-race}

5' RNA ligase-mediated rapid amplification of cDNA ends ( $5^{\prime}$-RLM-RACE) was done using a GeneRacer ${ }^{\text {Tix }}$ kit according to the manufacturer's instructions (Invitrogen, CA) but omitting the dephosphorylation and decapping steps. Briefly, $3 \mu \mathrm{g}$ of DNAse-treated total RNA was ligated to a GeneRacer Oligo RNA Adapter. First-strand cDNA was synthesized using oligo-dT. Specific primers were used to amplify $5^{\prime}$ ends by nested PCR from cDNA (Additional file 2: Table S1). The nested PCR products were separated on a $2 \%$ agarose gel, gel purified, ligated to a Zero Blunt TOPO vector (Invitrogen, CA) transformed into Topo 10 cells and sequenced to determine the cleavage site in target genes. Specific control were done using the specific primers.

\section{RNA-seq library sample preparation and sequencing}

Total RNA was extracted from rice leaves that had been treated or not with a $M$. oryzae spore solution following the whole-plant infection method $\left(10^{5}\right.$ spores $/ \mathrm{ml}, 0.2 \mathrm{ml} /$ plant, at 48 hpi) with the Maxwell 16 LEV Plant RNA Kit (Promega). Raw reads were checked for quality by using FastQC v0.11.3 (www.bioinformatics.babraham.ac. uk/projects/fastqc/) was used to check quality of raw reads; adapters were trimmed and removed with Trimmomatic v0.33 [87] (minimum quality score 35, minimum length 25). Reads obtained were mapped to the reference rice genome (MSU 7.0) provided with the reference gene annotation file (RGSP 7.0) by using STAR (v2.4.0j) [88]). Reads with mapping quality (MAPQ) $<30$ were removed. FeatureCounts (v1.4.5-p1) [89] was used to perform read summarization at the gene level, with the strand-specific option "reversely stranded". Statistical analysis of read counts was performed with $\mathrm{R}$, with the HTSFilter package [90] to remove low-expressed genes and the edge $R$ package [91] for differential expression analysis. To identify genes with significant difference in expression, a FDR cutoff $<0.05$ and $\log 2 \mathrm{FC} 1 \leq$ or $\geq 1$ was applied. Gene Ontology (GO) enrichment of differentially expressed genes involved Singular Enrichement analysis (SEA) using the AgriGO webtool $(p<0.01$ Fisher's test, TIGR genemodel) (http://bioinfo.cau.edu.cn/ agriGO/) [92]. Enriched GO terms were grouped, summarized and 2D-plotted by semantic clustering with the online analysis tool ReviGO (http://revigo.irb.hr/) [93].

\section{Quantification of Rice Diterpene Phytoalexins}

Leaf segments were collected from mock and $M$. oryzae-infected plants. Three biological replicates with two technical replicates each were performed. Approximately 200-300 $\mathrm{mg}$ of fresh plant material was soaked in $40 \mathrm{vol}$ of $70 \%$ methanol and incubated at $4{ }^{\circ} \mathrm{C}$ overnight with constant rotation. A $1 \mathrm{ml}$ aliquot was centrifuged at maximum speed to remove cell debris. Phytoalexins were quantified using $5 \mu \mathrm{l}$ of the extract by LC-MS/MS as described [94]. Significant differences in phytoalexin accumulation were evaluated with ANOVA.

\section{Supplementary information}

Supplementary information accompanies this paper at https://doi.org/10. 1186/s12870-019-2156-5.

Additional file 1: Figure S1. Characterization and phenotype of MIR7695-Ac and wild-type azygous (WT-Az) plants.

Additional file 2: Table S1. Oligonucleotides used in this study Additional file 3: Table S2. qPCR of T-DNA copy number in MIR7695Ac mutant plants with the sucrose phosphate synthase (SPS) gene as an endogenous reference.

Additional file 4: Figure S2.Resistance of MIR7695-Ac mutant plants to M. oryzae infection.

Additional file 5: Figure S3. Differentially expressed genes (DEGs) in leaves of MIR7695-Ac mutant plants relative to WT-Az plants, under noninfection or infection. 
Additional file 6: Table S3. Differentially expressed genes (DEGs) in MIR7695-Ac plants relative to wild-type azygous (WT-Az) plants (MIR7695Ac vs WT-Az).

Additional file 7: Table S4. Gene Ontology terms enriched in MIR7695Ac versus WT-Az plants according to molecular function.

Additional file 8: Table S5. DEGs in MIR7695-Ac versus WT-Az plants. Additional file 9: Figure S4. Validation of RNAseq data by qRT-PCR. Additional file 10: Table S6. DEGs in WT plants after infection with $M$. oryzae (48 h post-infection [hpi]) (INF-WT-Az vs. WT-Az).

Additional file 11: Table S7. DEGs in MIR7695-Ac plants after infection with M. oryzae (48 hpi) (INF-MIR7695-Ac vs. MIR7695-Ac).

Additional file 12: Table S8. Pathogenesis-related $(P R)$ gene expression in WT-Az and MIR7695-Ac plants during M. oryzae infection at $48 \mathrm{hpi}$.

Additional file 13: Table S9. DEGs in bifactorial analysis.

Additional file 14: Table S10. DEGs in the top GO categories that are overrepresented in MIR7695-Ac with M. oryzae infection (bifactorial analysis).

Additional file 15: Figure S5. Pathways for the biosynthesis of phenylpropanoids and diterpenoid phytoalexins in rice.

Additional file 16: Figure S6. RT-qPCR analysis of expression pattern of Fe homeostasis genes in WT-Az and MIR7695-Ac plants with M. oryzae infection.

\section{Abbreviations}

DEG: Differentially Expressed Gene; Fe: Iron; GO: Gene Ontology; LTP: Lipid Transfer protein; miR: MicroRNA; Nramp6: Natural Resistance-Associated Macrophage Pathogen 6; PR: Pathogenesis-Related; R: Resistance; ROS: Reactive Oxygen Species; TF: Transcription Factor

\section{Acknowledgements}

Not applicable

\section{Authors' contributions}

Conceived and designed experiments: SC, BSS, FSS, KO, YIH. Performed experiments: FSS, CPP, ST, SC; Analysed data: SC, FSS, BSS, KO, YIH. Wrote and revised manuscript: SC, BSS, KO, YIH. All authors read and approved the final manuscript.

\section{Funding}

This work was supported by the Spanish Ministry of Economy and Competitiveness (MINECO, BIO2015-67212-R) to BSS, the CSIC/NSC (Spanish Research Council/National Science Council of Taiwan)-Formosa Program (2009TW0041 and NSC 99-2923-B-001-002-MY3), the Academia Sinica Summit Project to $\mathrm{YICH}$, and the Japan Society for the Promotion of Science (JSPS KAKENHI, $17 \mathrm{H} 03811$ to KO). The funders have no role in the design of the study; collection, analysis and interpretation of data; and in writing the manuscript. We acknowledge support from the CERCA Programme ("Generalitat de Catalunya") and the "Severo Ochoa Programme for Centres of Excellence in R\&D" (MINECO, 2016-2019, SEV-2015-0533).

\section{Availability of data and materials}

The RNA sequence datasets generated during the current study will be available after May 2020 at the National Center for Biotechnology Information (NCBI) Gene Expression Omnibus (GEO) with the GSE122258 accession number (https://www.ncbi.nlm.nih.gov/geo/query/acc.cgi?acc= GSE122258). Until this date, the datasets are available from the corresponding author on reasonable request.

\section{Ethics approval and consent to participate}

Not applicable

\section{Consent for publication}

Not applicable

\section{Competing interests}

The authors declare that they have no competing interests.

\section{Author details}

${ }^{1}$ Centre for Research in Agricultural Genomics (CRAG) CSIC-IRTA-UAB-UB, Campus Universitat Autònoma de Barcelona (UAB), Bellaterra (Cerdanyola del Vallés), Barcelona, Spain. ${ }^{2}$ Biotechnology Research Center, The University of Tokyo, Tokyo, Japan. ${ }^{3}$ Institute of Plant and Microrbial Biology, Academia Sinica, Taipei, Taiwan. ${ }^{4}$ Consejo Superior de Investigaciones Científicas (CSIC), Barcelona, Spain.

Received: 26 February 2019 Accepted: 21 November 2019

Published online: 18 December 2019

\section{References}

1. Jones JDG, Dangl JL. The plant immune system. Nature. 2006;444:323-9.

2. Doughari J. An overview of plant immunity. J Plant Pathol Microbiol. 2015:6:1-11.

3. Boller T, Felix G. A renaissance of elicitors: perception of microbe-associated molecular patterns and danger signals by pattern-recognition receptors. Annu Rev Plant Biol. 2009;60:379-406.

4. Macho AP, Zipfel C. Plant PRRs and the activation of innate immune signaling. Mol Cell. 2014;54:263-72.

5. Cui H, Tsuda K, Parker JE. Effector-triggered immunity: from pathogen perception to robust defense. Annu Rev Plant Biol. 2015;66:487-511.

6. Li Y, Zhang Q, Zhang J, Wu L, Qi Y, Zhou J-M. Identification of MicroRNAs involved in pathogen-associated molecular pattern-triggered plant innate immunity. Plant Physiol. 2010;152:2222-31.

7. Navarro L, Dunoyer P, Jay F, Arnold B, Dharmasiri N, Estelle M, et al. A plant miRNA contributes to antibacterial resistance by repressing Auxin signaling. Science. 2006;312:436-9.

8. Shivaprasad PV, Chen H-M, Patel K, Bond DM, Santos BACM, Baulcombe DC. A MicroRNA superfamily regulates nucleotide binding site-Leucine-rich repeats and other mRNAs. Plant Cell. 2012;24:859-74.

9. Boccara M, Sarazin A, Thiébeauld O, Jay F, Voinnet O, Navarro L, et al. The Arabidopsis miR472-RDR6 silencing pathway modulates PAMP- and effector-triggered immunity through the post-transcriptional control of disease resistance genes. PLoS Pathog. 2014;10:e1003883.

10. Liu J, Cheng X, Liu D, Xu W, Wise R, Shen Q-H. The miR9863 family regulates distinct Mla alleles in barley to attenuate NLR receptor-triggered disease resistance and cell-death signaling. PLoS Genet. 2014;10:e1004755.

11. Li F, Pignatta D, Bendix C, Brunkard JO, Cohn MM, Tung J, et al. MicroRNA regulation of plant innate immune receptors. Proc Natl Acad Sci U S A. 2012;109:1790-5.

12. Jones-Rhoades M, Bartel D, Bartel B. MicroRNAs and their regulatory roles in plants. Annu Rev Plant Biol. 2006:57:19-53.

13. de Lima JC, Loss-Morais G, Margis R. MicroRNAs play critical roles during plant development and in response to abiotic stresses. Genet Mol Biol. 2012;35:1069-77.

14. Li C, Zhang B. MicroRNAs in control of plant development. J Cell Physiol. 2016:231:303-13.

15. Baldrich P, San SB. MicroRNAs in Rice innate immunity. Rice. 2016;9:1-6.

16. Chen X. A MicroRNA as a translational repressor of APETALA2 in Arabidopsis flower development. Science. 2004;303:2022-5.

17. Paul S, Datta SK, Datta K. miRNA regulation of nutrient homeostasis in plants. Front Plant Sci. 2015;6:232.

18. Chiou T-J, Aung K, Lin S-I, Wu C-C, Chiang S-F, Su C-L. Regulation of phosphate homeostasis by MicroRNA in Arabidopsis. Plant Cell. 2006; 18:412-21.

19. Liang G, Yang F, Yu D. MicroRNA395 mediates regulation of sulfate accumulation and allocation in Arabidopsis thaliana. Plant J. 2010;62:1046-57.

20. Abadía J. Leaf responses to Fe deficiency: a review. J Plant Nutr. 1992;15: 1699-713.

21. Naranjo-Arcos MA, Bauer P. Iron Nutrition, Oxidative Stress, and Pathogen Defense. In: Erkekoglu P, Kocer-Gumusel B, editors. Nutritional Deficiency. Rijeka: InTechOpen; 2016. p. 63-98.

22. Dangol S, Chen Y, Hwang BK, Jwa N-S. Iron- and reactive oxygen speciesdependent Ferroptotic cell death in Rice-Magnaporthe oryzae interactions. Plant Cell. 2019:31:189-209.

23. Torres MA. ROS in biotic interactions. Physiol Plant. 2010;138:414-29.

24. Huang H, Ullah F, Zhou D-X, Yi M, Zhao Y. Mechanisms of ROS regulation of plant development and stress responses. Front Plant Sci. 2019;10:800.

25. Marcec MJ, Gilroy S, Poovaiah BW, Tanaka K. Mutual interplay of $\mathrm{Ca}^{2+}$ and ROS signaling in plant immune response. Plant Sci. 2019;283:343-54. 
26. Waters BM, McInturf SA, Stein RJ. Rosette iron deficiency transcript and microRNA profiling reveals links between copper and iron homeostasis in Arabidopsis thaliana. J Exp Bot. 2012;63:5903-18.

27. Kong $W W$, Yang ZM. Identification of iron-deficiency responsive microRNA genes and cis-elements in Arabidopsis. Plant Physiol Biochem. 2010;48:153-9.

28. Valdés-López O, Yang SS, Aparicio-Fabre R, Graham PH, Reyes JL, Vance CP, et al. MicroRNA expression profile in common bean (Phaseolus vulgaris) under nutrient deficiency stresses and manganese toxicity. New Phytol. 2010;187:805-18

29. Agarwal S, Mangrauthia SK, Sarla N. Expression profiling of iron deficiency responsive microRNAs and gene targets in rice seedlings of Madhukar $\mathrm{x}$ Swarna recombinant inbred lines with contrasting levels of iron in seeds. Plant Soil. 2015:396:137-50.

30. Paul S, Gayen D, Datta SK, Datta K. Analysis of high iron rice lines reveals new miRNAs that target iron transporters in roots. J Exp Bot. 2016;67:5811-24.

31. Yu J, Hu S, Wang J, Wong GK-S, Li S, Liu B, et al. A draft sequence of the Rice genome (Oryza sativa L. ssp. indica). Science. 2002;296:79-92.

32. Goff SA, Ricke D, Lan T-H, Presting G, Wang R, Dunn M, et al. A draft sequence of the Rice genome (Oryza sativa L. ssp. japonica). Science. 2002;296:92-100.

33. Wilson RA, Talbot NJ. Under pressure: investigating the biology of plant infection by Magnaporthe oryzae. Nat Rev Microbiol. 2009;7:185-95.

34. Miura K, Ikeda M, Matsubara A, Song X-J, Ito M, Asano K, et al. OsSPL14 promotes panicle branching and higher grain productivity in rice. Nat Genet. 2010;42:545-9.

35. Wang S, Wu K, Yuan Q, Liu X, Liu Z, Lin X, et al. Control of grain size, shape and quality by OsSPL16 in rice. Nat Genet. 2012;44:950-4.

36. Zhang Y-C, Yu Y, Wang C-Y, Li Z-Y, Liu Q, Xu J, et al. Overexpression of microRNA OsmiR397 improves rice yield by increasing grain size and promoting panicle branching. Nat Biotechnol. 2013;31:848-52.

37. Fang Y, Xie K, Xiong L. Conserved miR164-targeted NAC genes negatively regulate drought resistance in rice. J Exp Bot. 2014;65:2119-35.

38. Yang W, Fan T, Hu X, Cheng T, Zhang M. Overexpressing Osa-miR171C decreases salt stress tolerance in rice. J Plant Biol. 2017;60:485-92.

39. Wang S, Sun X, Hoshino Y, Yu Y, Jia B, Sun Z, et al. MicroRNA319 positively regulates cold tolerance by targeting OsPCF6 and OsTCP21 in Rice (Oryza sativa L.). PLoS One. 2014;9:e91357.

40. Baldrich P, Campo S, Wu M-T, Liu T-T, Hsing Y-IC, Segundo BS. MicroRNAmediated regulation of gene expression in the response of rice plants to fungal elicitors. RNA Biol. 2015;12:847-63.

41. Campo S, Peris-Peris C, Siré C, Moreno AB, Donaire L, Zytnicki M, et al. Identification of a novel microRNA (miRNA) from rice that targets an alternatively spliced transcript of the Nramp6 (Natural resistance-associated macrophage protein 6) gene involved in pathogen resistance. New Phytol. 2013;199:212-27.

42. Li Y, Lu Y-G, Shi Y, Wu L, Xu Y-J, Huang F, et al. Multiple rice microRNAs are involved in immunity against the blast fungus Magnaporthe oryzae. Plant Physiol. 2014;164:1077-92.

43. Li Z-Y, Xia J, Chen Z, Yu Y, Li Q-F, Zhang Y-C, et al. Large-scale rewiring of innate immunity circuitry and microRNA regulation during initial rice blast infection. Sci Rep. 2016;6:25493.

44. Zhang X, Bao Y, Shan D, Wang Z, Song X, Wang Z, et al. Magnaporthe oryzae induces the expression of a MicroRNA to suppress the immune response in Rice. Plant Physiol. 2018;177:352-68.

45. Salvador-Guirao R, Hsing Y-I, San SB. The Polycistronic miR166k-166h positively regulates Rice immunity via post-transcriptional control of EIN2. Front Plant Sci. 2018;9:337.

46. Li Y, Zhao S-L, Li J-L, Hu X-H, Wang H, Cao X-L, et al. Osa-miR169 negatively regulates Rice immunity against the blast fungus Magnaporthe oryzae. Front Plant Sci. 2017:8:2

47. Wang Z, Xia Y, Lin S, Wang Y, Guo B, Song X, et al. Osa-miR164a targets OsNAC60 and negatively regulates rice immunity against the blast fungus Magnaporthe oryzae. Plant J. 2018;95:584-97.

48. Peris-Peris C, Serra-Cardona A, Campo S, Ariño J. Two NRAMP6 isoforms function as iron and manganese transporters and contribute to disease resistance in rice. Mol Plant-Microbe Interact. 2017;30:385-98.

49. Hsing YI, Chern CG, Fan MJ, Lu PC, Chen KT, Lo SF, et al. A rice gene activation/knockout mutant resource for high throughput functional genomics. Plant Mol Biol. 2007;63:351-64.

50. Wu H-P, Wei F-J, Wu C-C, Lo S-F, Chen L-J, Fan M-J, et al. Large-scale phenomics analysis of a T-DNA tagged mutant population. Gigascience. 2017;6:1-7.
51. Lo S-F, Fan M-J, Hsing Y-I, Chen L-J, Chen S, Wen I-C, et al. Genetic resources offer efficient tools for rice functional genomics research. Plant Cell Environ. 2016;39:998-1013.

52. Agrawal GK, Rakwal R, Jwa NS, Agrawal VP. Signalling molecules and blast pathogen attack activates rice OsPR1a and OsPR1b genes: a model illustrating components participating during defence/stress response. Plant Physiol Biochem. 2001;39:1095-103.

53. Wei T, Ou B, Li J, Zhao Y, Guo D, Zhu Y, et al. Transcriptional profiling of Rice early response to Magnaporthe oryzae identified OsWRKYs as important regulators in Rice blast resistance. PLoS One. 2013;8:e59720.

54. Shimono M, Koga H, Akagi A, Hayashi N, Goto S, Sawada M, et al. Rice WRKY45 plays important roles in fungal and bacterial disease resistance. Mol Plant Pathol. 2012;13:83-94.

55. Kaneda T, Taga Y, Takai R, Iwano M, Matsui H, Takayama S, et al. The transcription factor OsNAC4 is a key positive regulator of plant hypersensitive cell death. EMBO J. 2009;28:926-36.

56. Miyamoto K, Shimizu T, Mochizuki S, Nishizawa Y, Minami E, Nojiri H, et al. Stress-induced expression of the transcription factor RERJ1 is tightly regulated in response to jasmonic acid accumulation in rice. Protoplasma. 2013;250:241-9.

57. Inoue $H$, Higuchi K, Takahashi M, Nakanishi H, Mori S, Nishizawa NK. Three rice nicotianamine synthase genes, OsNAS1, OsNAS2, and OsNAS3 are expressed in cells involved in long-distance transport of iron and differentially regulated by iron. Plant J. 2003;36:366-81.

58. Ali S, Ganai BA, Kamili AN, Bhat AA, Mir ZA, Bhat JA, et al. Pathogenesisrelated proteins and peptides as promising tools for engineering plants with multiple stress tolerance. Microbiol Res. 2018;212-213:29-37.

59. Galeotti F, Barile E, Curir P, Dolci M, Lanzotti V. Flavonoids from carnation (Dianthus caryophyllus) and their antifungal activity. Phytochem Lett. 2008;1:44-8.

60. Miedes E, Vanholme R, Boerjan W, Molina A. The role of the secondary cell wall in plant resistance to pathogens. Front Plant Sci. 2014;5:358.

61. Kawahara Y, Oono Y, Kanamori H, Matsumoto T, Itoh T, Minami E, et al. Simultaneous RNA-Seq analysis of a mixed Transcriptome of Rice and blast fungus interaction. PLoS One. 2012;7:e49423.

62. Wang Y, Kwon SJ, Wu J, Choi J, Lee Y-H, Agrawal GK, et al. Transcriptome analysis of early responsive genes in Rice during Magnaporthe oryzae infection. Plant Pathol J. 2014;30:343-54.

63. Huang L-F, Lin K-H, He S-L, Chen J-L, Jiang J-Z, Chen B-H, et al. Multiple patterns of regulation and overexpression of a Ribonuclease-like pathogenesis-related protein gene, OsPR10a, Conferring Disease Resistance in Rice and Arabidopsis. PLoS One. 2016;11:e0156414.

64. Pereira MP, Santos C, Gomes A, Vasconcelos MW. Cultivar variability of iron uptake mechanisms in rice (Oryza sativa L.). Plant Physiol Biochem. 2014;85:21-30.

65. Kobayashi T, Nishizawa NK. Iron uptake, translocation, and regulation in higher plants. Annu Rev Plant Biol. 2012;63:131-52.

66. Ahuja I, Kissen R, Bones AM. Phytoalexins in defense against pathogens. Trends Plant Sci. 2012;17:73-90.

67. Hasegawa M, Mitsuhara I, Seo S, Imai T, Koga J, Okada K, et al. Phytoalexin accumulation in the interaction between Rice and the blast fungus. Mol Plant-Microbe Interact. 2010;23:1000-11.

68. Umemura K, Ogawa N, Shimura M, Koga J, Usami H, Kono T. Possible role of Phytocassane, Rice Phytoalexin, in disease resistance of Rice against the blast fungus Magnaporthe grisea. Biosci Biotechnol Biochem. 2003;67:899-902.

69. Dillon VM, Overton J, Grayer RJ, Harborne JB. Differences in phytoalexin response among rice cultivars of different resistance to blast. Phytochemistry. 1997;44:599-603.

70. Hood MI, Skaar EP. Nutritional immunity: transition metals at the pathogenhost interface. Nat Rev Microbiol. 2012;10:525-37.

71. Pereira EG, Oliva MA, Rosado-Souza L, Mendes GC, Colares DS, Stopato CH, et al. Iron excess affects rice photosynthesis through stomatal and nonstomatal limitations. Plant Sci. 2013;201-202:81-92.

72. Liu G, Greenshields DL, Sammynaiken R, Hirji RN, Selvaraj G, Wei Y. Targeted alterations in iron homeostasis underlie plant defense responses. J Cell Sci. 2007;120:596

73. Aznar A, Patrit O, Berger A, Dellagi A. Alterations of iron distribution in Arabidopsis tissues infected by Dickeya dadantii. Mol Plant Pathol. 2015; 16:521-8.

74. Ricachenevsky FK, Sperotto RA. There and back again, or always there? The evolution of rice combined strategy for Fe uptake. Front Plant Sci. 2014;5:189.

75. Conrath U, Beckers GJM, Langenbach CJG, Jaskiewicz MR. Priming for enhanced defense. Annu Rev Phytopathol. 2015;53:97-119. 
76. Selitrennikoff CP. Antifungal proteins. Appl Environ Microbiol. 2001;67:2883-94.

77. Almagro L, Gómez Ros LV, Belchi-Navarro S, Bru R, Ros Barceló A, Pedreño MA. Class III peroxidases in plant defence reactions. J Exp Bot. 2009;60:377-90.

78. Cho M-H, Lee S-W. Phenolic Phytoalexins in Rice: biological functions and biosynthesis. Int J Mol Sci. 2015;16:29120.

79. Dixon RA, Achnine L, Kota P, Liu C-J, Reddy MSS, Wang L. The phenylpropanoid pathway and plant defence-a genomics perspective. Mol Plant Pathol. 2002;3:371-90.

80. Camargo-Ramírez R, Val-Torregrosa B, San SB. MiR858-mediated regulation of flavonoid-specific MYB transcription factor genes controls resistance to pathogen infection in Arabidopsis. Plant Cell Physiol. 2018;59:190-204.

81. Murray MG, Thompson WF. Rapid isolation of high molecular weight plant DNA. Nucleic Acids Res. 1980;8:4321-6.

82. Yang L, Ding J, Zhang C, Jia J, Weng H, Liu W, et al. Estimating the copy number of transgenes in transformed rice by real-time quantitative PCR. Plant Cell Rep. 2005;23:759-63.

83. Roschzttardtz H, Conejero G, Curie C, Mari S. Identification of the endodermal vacuole as the Iron storage compartment in the Arabidopsis embryo. Plant Physiol. 2009;151:1329-38.

84. Sesma A, Osbourn AE. The rice leaf blast pathogen undergoes developmental processes typical of root-infecting fungi. Nature. 2004;431:582.

85. Coca M, Bortolotti C, Rufat M, Peñas G, Eritja R, Tharreau D, et al. Transgenic rice plants expressing the antifungal AFP protein from Aspergillus giganteus show enhanced resistance to the rice blast gungus Magnaporthe grisea. Plant Mol Biol. 2004;54:245-59.

86. Lamari L. Assess 2.0: image analysis software for plant disease quantification. St. Paul, MN: APS Press; 2008

87. Bolger AM, Lohse M, Usadel B. Trimmomatic: a flexible trimmer for Illumina sequence data. Bioinformatics. 2014;30:2114-20.

88. Dobin A, Davis CA, Schlesinger F, Drenkow J, Zaleski C, Jha S, et al. STAR: ultrafast universal RNA-seq aligner. Bioinformatics. 2013;29:15-21.

89. Liao Y, Smyth GK, Shi W. FeatureCounts: an efficient general purpose program for assigning sequence reads to genomic features. Bioinformatics. 2014;30:923-30.

90. Rau A, Gallopin M, Celeux G, Jaffrézic F. Data-based filtering for replicated high-throughput transcriptome sequencing experiments. Bioinformatics. 2013;29:2146-52

91. Robinson MD, McCarthy DJ, Smyth GK. edgeR: a bioconductor package for differential expression analysis of digital gene expression data. Bioinformatics. 2009;26:139-40.

92. Du Z, Zhou X, Ling Y, Zhang ZH, Su Z. AgriGO: a GO analysis toolkit for the agricultural community. Nucleic Acids Res. 2010;38:W64-70

93. Supek F, Bosnjak M, Skunca N, Smuc T. REVIGO summarizes and visualizes long lists of gene ontology terms. PLoS One. 2011;6:1-9.

94. Miyamoto K, Fujita M, Shenton MR, Akashi S, Sugawara C, Sakai A, et al. Evolutionary trajectory of phytoalexin biosynthetic gene clusters in rice. Plant J. 2016;87:293-304

\section{Publisher's Note}

Springer Nature remains neutral with regard to jurisdictional claims in published maps and institutional affiliations.

Ready to submit your research? Choose BMC and benefit from:

- fast, convenient online submission

- thorough peer review by experienced researchers in your field

- rapid publication on acceptance

- support for research data, including large and complex data types

- gold Open Access which fosters wider collaboration and increased citations

- maximum visibility for your research: over $100 \mathrm{M}$ website views per year

At $\mathrm{BMC}$, research is always in progress.

Learn more biomedcentral.com/submissions 